\title{
hnRNPH1 recruits PTBP2 and SRSF3 to cooperatively modulate alternative pre-mRNA splicing in germ cells and is essential for spermatogenesis and oogenesis
}

\section{Shuiqiao Yuan ( $\nabla$ shuiqiaoyuan@hust.edu.cn )}

Huazhong University of Science and Technology https://orcid.org/0000-0003-1460-7682

\section{Shenglei Feng}

Huazhong University of Science and Technology

Jinmei Li

Huazhong University of Science and Technology

Hui Wen

Huazhong University of Science and Technology

Kuan Liu

Huazhong University of Science and Technology

Yiqian Gui

Huazhong University of Science and Technology

\section{Yujiao Wen}

Huazhong University of Science and Technology

\section{Xiaoli Wang}

Huazhong University of Science and Technology

\section{Article}

Keywords: hnRNPH1, Alternative splicing, Spermatogenesis, Oogenesis, Mice

Posted Date: November 11th, 2021

DOI: https://doi.org/10.21203/rs.3.rs-1060705/v1

License: (c) (i) This work is licensed under a Creative Commons Attribution 4.0 International License. Read Full License

Version of Record: A version of this preprint was published at Nature Communications on June 23rd, 2022. See the published version at https://doi.org/10.1038/s41467-022-31364-7. 


\section{Abstract}

Coordinated regulation of alternative pre-mRNA splicing is essential for germ cell development. However, the molecular mechanism underlying that control alternative mRNA expression during germ cell development remains poorly understood. Herein, we showed that hnRNPH1, an RNA-binding protein, is highly expressed in the reproductive system and localized in the chromosomes of meiotic cells but excluded from the XY body in pachytene spermatocytes and recruits the splicing regulators PTBP2 and SRSF3 and cooperatively regulates the alternative splicing of the critical genes that are required for spermatogenesis. Conditional knockout Hnrnph1 in spermatogenic cells caused many abnormal splicing events that affect genes related to meiosis and communication between germ cells and Sertoli cells, characterized by asynapsis of chromosomes and impairment of germ-Sertoli communications, ultimately leading to male sterility. We further showed that hnRNPH1 could directly bind to SP011 and recruit the splicing regulators PTBP2 and SRSF3 to regulate the alternative splicing of the target genes cooperatively. Strikingly, Hnrnph1 germline-specific mutant female mice were also infertile, and Hnrnph1deficient oocytes exhibited a similar defective synapsis and cell-cell junction as shown in Hnrnph1deficient male germ cells. Collectively, our data reveal an essential role for hnRNPH1 in regulating premRNA splicing during spermatogenesis and oogenesis and support a molecular model whereby hnRNPH1 governs a network of alternative splicing events in germ cells via recruiting PTBP2 and SRSF3.

\section{Introduction}

In mammals, male germ cells undergo self-renewal of spermatogonial stem cells, proliferation and differentiation of spermatogonia, genomic rearrangement of homologous recombination at meiosis, morphological changes from round spermatids to elongated spermatids, and finally gives rise to mature spermatozoa ${ }^{1}$. Numerous high-throughput sequencing has revealed the important role of alternative premRNA splicing in the transcriptome and proteome diversification during spermatogenesis ${ }^{2}$. Most of the genes involved in spermatogenesis are transcribed and processed into multiple isoforms, mainly through alternative splicing that can expand the form and function of the genome with limited gene number and is especially important for highly complex organisms such as testis ${ }^{3}$. An interesting example is represented by the Spo11 gene, encoding for two main protein isoforms (SP011a and $\beta$ ) and their corresponding transcripts differ for exon 2 skipping (a) or inclusion ( $\beta$ ). Early meiotic spermatocytes mainly produce SP011 $\beta$, whereas SP011 a becomes predominant in late meiosis ${ }^{4,5}$. Strikingly, the timing of Spo11 alternative splicing parallels that of DSB formation during meiosis, with a first wave that occurs on autosomal chromosomes in leptotene-to-zygotene spermatocytes and a delayed one that switches to sex chromosomes in late pachytene spermatocytes. Transgenic mice expressing only SPO11 $\beta$ were fully competent in establishing the first DSB wave, but late foci in sex chromosomes executed by SP011a were suppressed, leading to male sterility due to inefficient $X Y$ pairing and recombination ${ }^{6}$. In addition, other genes with alternative splicing to generate different transcripts encoding numerous protein isoforms were identified, such as $C-k i t^{7}$, Spata $^{8}, C^{2}{ }^{9} m^{9}, A c r b p^{10}$ and $Y b \times 3^{11}$. Nevertheless, not all spliced variant seems to encode functional proteins. For example, the full-length Sox17gene highly expressed in 
spermatogonia encodes a transcription factor with a high mobility group (HMG) box region in its $\mathrm{N}$ terminus, while an alternatively spliced variant $t$-Sox 17 in pachytene spermatocytes encodes a truncated protein lacking the entire HMG box region due to the skipping exon and is unable to bind DNA to stimulate transcription ${ }^{12}$.

Pre-mRNA splicing is mediated by splicing factors, including the large ribonucleoprotein (RNP) complex known as the spliceosome and particular RNA-binding proteins facilitating the regulation of spliceosome assembly and splice site usage ${ }^{13}$. A growing number of novel splicing regulators involved in spermatogenesis continue to be uncovered by using mouse knockout models, such as SAM68, MRG15, PTBP2, RBM5, and BCAS2 ${ }^{14-18}$. Notably, PTBP2 could also to control a network of genes involved in cell adhesion and polarity and was essential for Sertoli-germ cell communications ${ }^{19}$. However, the underlying mechanisms of how alternative pre-mRNA splicing functions in germ cell development remain poor understood.

Heterogeneous nuclear ribonucleoproteins (hnRNPs) are polyvalent RNA-binding proteins with crucial roles in multiple aspects of RNA metabolism, including alternative splicing, mRNA decay, the packaging of nascent transcripts, and translational regulation ${ }^{20,21}$. Some members of the hnRNP family were reported to be highly expressed in male germ cells and involved in the process of spermatogenesis ${ }^{22,23}$. In addition, hnRNP G-T, as an efficient spermatogenic cell-specific splicing factor, can cooperate with RBMY to modulate the signaling pathways in the testis ${ }^{24}$. Among the hnRNP family members, hnRNPH1 has attracted increasing public attention based on its essential role in neurological diseases and cancers $^{25-27}$. However, its physiological function and potential mechanism in the reproductive system remain still unknown.

Here, we find that hnRNPH1 is highly expressed in meiotic cells and is required for pre-mRNA alternative splicing and spermatogenesis. Disruption of hnRNPH1 in male germ cells causes the abnormal alternative mRNA splicing and affects the meiosis and germ-Sertoli cell communications, which ultimately leads to male sterility. We further show that hnRNPH1 could directly bind to SPO11 and recruit the splicing regulators PTBP2 and SRSF3 to cooperatively regulate the alternative splicing of the target genes. Strikingly, we find that hnRNPH1 is also essential for oogenesis, and depletion of hnRNPH1 in embryonic female germ cells leads to female infertility with a similar defect of meiosis and cell-cell junction as shown in hnRNPH1-deficient male germ cells. Our data demonstrate a critical role of hnRNPH1 in pre-mRNA alternative splicing of both male and female germ cells and fertility.

\section{Results}

\section{hnRNPH1 is highly expressed in meiotic cells and localizes to the chromosomes}


To explore whether hnRNPH1 plays a role in the reproductive system, we first characterized its spatiotemporal expression pattern. We observed high Hnrnph1 mRNA and protein expression levels in both the testis and ovary (Supplementary Fig.S1a-b). Interestingly, both Hnrnph1 mRNA and protein expression levels were gradually increased and maintained at a high level from P14 testes (Supplementary Fig.S1c-d). We then detected the subcellular localization of hnRNPH1 in the adult testis by immunofluorescence (IF) and found hnRNPH1 was mainly expressed in spermatocytes, round spermatids, and Sertoli cells but was absent in spermatogonia and elongated spermatids (Fig. 1a and Supplementary Fig.S1e), suggesting hnRNPH1 might function in meiotic processes. We thus examined the detailed localization of hnRNPH1 during meiosis through chromosome spreads to infer the specific stage at which hnRNPH1 functions. Consequently, the expression of hnRNPH1 was weak in leptotene and zygotene stages, began to be increasing in the pachytene stage, and maintained the highest level from mid pachytene to diplotene stage, but was not observed in metaphase (Fig. 1b and c). Interestingly, hnRNPH1 was found to be excluded from the XY body formed in pachytene stage (Fig. 1c). Next, we examined the localization of hnRNPH1 in meiotic chromosomes of oocytes and found a consistent localization pattern with spermatogenic cells (Supplementary Fig.S1f-g). These results suggest a conserved function of hnRNPH1 in both male and female meiosis during germ cell development.

\section{hnRNPH1 interplays with mRNA splicing factors PTBP2 and SRSF3 in testes}

To elucidate the physiological functions of hnRNPH1 in germ cell development, we performed immunoprecipitation-mass spectrometry (IP-MS) using hnRNPH1 antibody to unbiasedly identify the interactome of hnRNPH1 in wild-type (WT) testes. Consequently, a total of 171 proteins were identified from the hnRNPH1 antibody pull-down products (Fig. 2a and Supplementary Table 1). Gene Ontology (GO) and KEGG enrichment analyses revealed that 17 out of those proteins are related to mRNA splicing (Fig. 2b). Interestingly, among the $16 \mathrm{hnRNPH} 1$ interacting proteins screened by the STRING database (Fig. 2c), three key splicing factors, PTBP2 ${ }^{28}, \mathrm{SRSF}^{29}$, and SNRNP70 ${ }^{14}$, have been reported to be directly or indirectly involved in spermatogenesis. Through co-immunoprecipitation (Co-IP) assays, we further confirmed that PTBP2 and SRSF3 interacted with hnRNPH1 in the testis (Fig. 2d), while the interaction of SNRNP70 with hnRNPH1 seems to depend on RNA (Supplementary Fig.S2a). In addition, IF assay revealed that an obvious co-localization of hnRNPH1 with PTBP2 and SRSF3 was observed in spermatocytes and round spermatids (Fig. 2e), which further supported the interaction effects of hnRNPH1 with PTBP2 and SRSF3 in male germ cells. More interestingly, highly consistent with hnRNPH1, both PTBP2 and SRSF3 showed an increased expression from the pachytene stage and were excluded from the XY body (Supplementary Fig. S2b).

To further understand the potential mechanism by which hnRNPH1 interacts with PTBP2 and SRSF3 in vitro, we examined their direct association among hnRNPH1, PTBP2, and SRSF3 by ectopically coexpressed hnRNPH1, PTBP2, and SRSF3 in HEK293T cells. Reciprocal Co-IP assays showed that hnRNPH1 could directly bind with PTBP2 and SRSF3 (Fig. 2f-i). We further found that the 200-288aa 
region (containing the GRY domain) of hnRNPH1 was necessary for binding to the 50-180aa region (containing the RRM1 domain) of PTBP2 (Fig. 2f-g) and the 100-200aa region (containing the qRRM2 domain) of hnRNPH1 was responsible for interaction with the 86-164aa region (containing the RS domain) of SRSF3 (Fig. $2 \mathrm{~h}-\mathrm{i}$ ). These findings suggest that hnRNPH1 cooperates with mRNA splicing factors PTBP2 and SRSF3 through specific regions.

\section{hnRNPH1 is required for embryonic development, spermatogenesis and male fertility}

To further determine the physiological roles of hnRNPH1 in the reproductive system, we first generated Hnrnph1 global knockout mouse line (herein called hnRNPH1 gKO) by crossing germ-line Cre deleter mice with Hnrnph 1-floxed mice (see the details in materials and methods section) (Supplementary Fig.S3a-b). Surprisingly, we found that hnRNPH1 gKO mice could not develop to full-term birth and died at 13.5 days of the embryonic stage (Fig. 3a and Supplementary Fig.S3c-e), suggesting that hnRNPH1 is required for mouse embryonic development. These results hinder us from investigating the physiological functions of hnRNPH1 in the reproductive system. We then created germline conditional Hnrnph1 knockout mice by utilizing the Stra8-GFPCre knock-in mouse line ${ }^{30}$ to delete exon 6 of the Hnrnph1 gene in germ cells (Supplementary Fig.S3a-b). Stra8-GFPCre induces recombination starting from type A1 spermatogonia in males (before meiosis) and embryonic ovarian germ cells in females (embryonic day $12.5)^{30,31}$. Western blot (WB), RT-qPCR, and IF analyses showed a significant decrease of both Hnrnph1 mRNA and protein expression levels in conditional knockout mouse (Hnrnph $1^{\text {flox/Del }}$ Stra8-GFPCre, hereafter referred to as hnRNPH1 CKO) testes compared with that of controls (Hnrnph $7^{\text {flox/flox }}$ or Hnrnph1 ${ }^{+/ \text {flox }}$ Stra8-GFPCre, hereafter called control) (Fig. 3b-d), suggesting that we successfully generated germ line-specific hnRNPH1 mutants. Interestingly, the hnRNPH1 ablation did not affect the expression of its homolog hnRNPF in testes (Fig. 3b-c) and the localization of its interacting partners PTBP2 and SRSF3 in testes (Fig. 3e-f).

Although hnRNPH1 cKO males were viable and appeared to be grossly normal, they exhibited completely infertile in 5-month-long fertility tests. Testis size from hnRNPH1 cKO males was significantly smaller than their littermate controls (Fig. 3g). The testis/body weight ratio of hnRNPH1 cKO males decreased significantly compared with the control group from postnatal day 28 (P28) onward (Fig. 3h). Histological analyses showed that in adult hnRNPH1 $\mathrm{cKO}$ males, there is no detectable mature sperm in seminiferous tubules at stages VII-VIII (Fig. 3i), and spermatogenesis completely arrested at in step 15 spermatids (Supplementary Fig.S4a). In comparison to controls, the apoptotic cells in hnRNPH1 cKO testes were found to be increased significantly by TUNEL assay even at the early P18 stage, suggesting that the development of spermatocytes may have defect during the first wave of spermatogenesis (Supplementary Fig.S4b). Interestingly, although round spermatids were histologically normal in hnRNPH1 cKO testes, some of them were prematurely sloughed into epididymis even at P24 (Fig. 3i-j). In addition, no abnormalities were observed in the development of early male germ cells in hnRNPH1 cKO testes (Supplementary Fig.S4c-d), including undifferentiated spermatogonia $\left(\mathrm{PLZF}^{+}\right)$and pre-meiotic 
germ cells $\left(\mathrm{STRA}^{+}\right)$. These results indicate that hnRNPH1 is indispensable for spermatogenesis in mice and that deletion of hnRNPH1 in spermatogenic cells results in male sterility.

\section{Ablation of hnRNPH1 leads to aberrant mRNA splicing events in male germ cells}

Considering that hnRNPH1 is mainly confined to the nucleus of germ cells and interacts with key splicing factors PTBP2 and SRSF3, we speculated that hnRNPH1 is involved in regulating alternative splicing in male germ cells. To test this possibility, we performed high-throughput RNA-seq using isolated meiotic pachytene spermatocytes and post-meiotic round spermatids from adult hnRNPH1 cKO and control testes. As we expected, the RNA-seq analysis identified a large number of mRNA splicing changes in both hnRNPH1 cKO spermatocytes (Supplementary Table 2) and round spermatids (Supplementary Table 3), including skipped exons (SE), alternative 5' splice sites (A5SS), alternative 3' splice sites (A3SS), mutually exclusive exons (MXE), and retained introns (RI) (Fig. 4a; $\mid \triangle P S \|>10 \%, P$-value $<0.05$ ), affecting a total of $\sim 8,00$ genes respectively. About $55.8 \%$ and $60.1 \%$ of AS events were found to be upregulated in hnRNPH1 cKO spermatocytes and round spermatids, respectively (Fig. 4b), suggesting the splicing events repressed by hnRNPH1 were slightly more than those promoted by hnRNPH1 during spermatogenesis. Interestingly, we found SE was the predominant splicing type in both pachytene spermatocytes $(79.6 \%)$ and round spermatids (76.3\%) among the alternative splicing events affected by hnRNPH1 ablation (Fig. 4c). In addition, hierarchical clustering of differentially expressed genes (DEG) showed that, in pachytene spermatocytes, a total of 3283 genes were upregulated ( 91.4\%) and 310 genes were downregulated $(\sim 8.6 \%)$ in hnRNPH1 cKO mice compared to that of control mice. In round spermatids, a total of 2892 genes were upregulated $(\sim 2.8 \%)$ and 599 genes were downregulated $(\sim 17.2 \%)$ in hnRNPH1 cKO mice compared to that of control mice (Supplementary Fig.S5a-b and Supplementary Table 4-5; $P$-value $<0.05$, fold change $>2$ ). These data suggest that hnRNPH 1 could affect the transcriptional levels by repressing the gene expression to a large extent during spermatogenesis. Notably, we found that the alternative splicing altered genes in hnRNPH1 cKO pachytene spermatocytes and round spermatids showed an extensive overlap (Fig. 4d); however, whether in pachytene spermatocytes or round spermatids, comparison between the differential expression genes and the alternative splicing changed genes displayed a limited overlap (Supplementary Fig.S5c), suggesting distinct regulation mechanisms of hnRNPH1 in transcription and splicing.

Given that PTBP2 has been reported to regulate the mRNA alternative splicing during spermatogenesis and the ability of hnRNPH1 to interact with PTBP2 (Fig. 2), we, therefore, asked whether hnRNPH1 could regulate the same alternative splicing genes with PTBP2. To this end, we reanalyzed the published RNAseq data from Ptbp2 cKO mice ${ }^{19}$ to ensure a consistent analysis criterion with our RNA-seq data analysis of hnRNPH1 cKO mice ( Supplementary Table 6). Of note, in PTBP2 null testes, SE also accounts for the most significant proportion (83.2\%) among 5 alternative splicing types (Supplementary Fig.S5d). Further analysis revealed that hnRNPH1 and PTBP2 share many alternative splicing genes (Fig. 4d) and events (Fig. 4e) in pachytene spermatocytes and round spermatids. Interestingly, GO term analysis of their shared alternative splicing event-regulated genes highlighted the specific enrichment in functional 
categories involved in germ cell development, such as "synapsis" and "spermatogenesis" in pachytene spermatocytes (Fig. 4f), as well as "fertilization" and "adhesion junction" in round spermatids (Fig. 4g). Despite poor correlations $(R=-0.024$ in pachytene spermatocytes and $R=0.023$ in round spermatids) of $\triangle \mathrm{PSI}$ values of shared alternative splicing event-regulated genes between hnRNPH1 cKO spermatogenic cells (both spermatocytes and spermatids) and Ptbp2 cKO testes (Fig. 4h-i), most of the genes involved in germ cell development were regulated in the same direction. Notably, only a few of those genes showed abnormal expression levels in hnRNPH1 cKO spermatogenic cells (Supplementary Fig.S5e-f), especially in spermatids. Taken together, these analyses indicate that hnRNPH1 and PTBP2 could co-regulate the mRNA splicing of some essential genes related to spermatogenesis.

Since the transition from male meiotic spermatocytes to post-meiotic round spermatids is accompanied by genome-wide reprogramming of splicing ${ }^{2}$, we next wanted to ask whether the genome-wide reprogramming of splicing was affected upon hnRNPH1 deletion during this transition process. By analyzing RNA-seq data, we found that a number of alternative splicing genes or events related to developmental regulation during meiotic spermatocytes to post-meiotic round spermatids transition overlapped with those susceptible to hnRNPH1 ablation in pachytene spermatocytes and round spermatids (Supplementary Fig.S6a), and the $\triangle P S I$ values showed a highly positive correlation $(R=0.68$, $P=2.2^{e-16}$ ) (Supplementary Fig.S6b). Remarkably, we found that both the protein and mRNA levels of hnRNPH1 progressively declined from pachytene spermatocytes to round spermatids during spermatogenesis (Supplementary Fig.S6c-d). Together, these data suggest that hnRNPH1 may be involved in the genome-wide reprogramming of splicing during meiotic to post-meiotic transition, and its change in expression may contribute to the timely regulated selection of specific alternative splicing events across meiosis.

\section{Lack of hnRNPH1 in male germ cells disrupts meiotic processes}

Because some alternative splicing genes related to meiosis, such as synapsis and DNA recombination, were affected in hnRNPH1 cKO spermatocytes (Fig. 4f), we determined whether the splicing levels of corresponding exons of those genes were altered in isolated pachytene spermatocytes. RT-PCR analysis of 10 selected alternative splicing genes most closely related to meiosis yielded an $60 \%$ validation rate (6/10) (Fig. 5a-b and Supplementary Fig.S7a), indicating a relative reliability of the RNA-seq data. WB results further revealed a significant change of protein expression levels of some meiosis-related genes (e.g. Smc2, Cpeb1, Ehmt2, and Brme1) in hnRNPH1 cKO spermatocytes compared with controls (Fig. 5c), suggesting that hnRNPH1 ablation may affect the meiosis process by regulating some meiosis-related gene expression in transcriptional and translational levels.

To evaluated the abnormality of hnRNPH1 cKO spermatocytes during meiosis, we checked the meiotic processes by chromosome spreading analyses. In control pachytene spermatocytes, with DSB repair completed, $\mathrm{YH} 2 \mathrm{AX}$ signals were removed from autosomes but confined to the sex chromosomes. However, a higher proportion of hnRNPH1 cKO pachytene (29.6\% in cKO vs. $3.8 \%$ in control) and 
diplotene $(20.5 \%$ in cKO vs. $4.6 \%$ in control) spermatocytes displayed an abnormal $\mathrm{Y}-\mathrm{H} 2 \mathrm{~A} . \mathrm{X}$ distribution compared with controls (Fig. $5 \mathrm{~d}-\mathrm{e}$ ), indicating that DNA damage response remained active in synapsed homologs of hnRNPH1-null spermatocytes. Moreover, developmental retardation was observed in hnRNPH1 cKO spermatocyte characterized by a lower proportion of pachytene and diplotene spermatocytes than that of controls (Fig. 5f). At the pachytene stage, each pair of homologous autosomes complete synapsis, while the pairing of sex chromosomes in males only occurs at a small region, known as the pseudoautosomal region (PAR). SYCP1, an important component of the synaptic complex, was used as a marker for synapsis. Interestingly, SYCP1 was present at the PAR between XY chromosomes in control pachynema, while three types of abnormal asynapsis were observed in hnRNPH1 cKO pachynema, including autosomal asynasis ( $9.2 \%$ in cKO vs. $3.6 \%$ in control), mislocalization of SYCP1 to unsynapsed sex chromosomes (WT=2.9\%; $14.0 \%$ in cKO vs. $2.9 \%$ in control), and separated X-Y chromosomes (34.6\% in cKO vs. 3.3\% in control) (Fig. 5g-h). In addition, although hnRNPH1 deletion did not affect the total number of MLH1 foci (a crossover marker) in pachytene spermatocytes, nearly $30 \%$ of the pachytene spermatocytes did not form crossovers on sex chromosomes due to separation of X-Y (Supplementary Fig.S7b-c). Taken together, these results indicate that hnRNPH1 ablation caused chromosomes asynapsis,especially the high level of unpaired sex chromosomes.

Given that previous studies reported that unpaired sex chromosomes in male mice could be caused by abnormal Spo11 pre-mRNA splicing ${ }^{32}$ and hnRNPH1 was identified as a key regulator of Spo11a splicing in mouse spermatocytes ${ }^{33}$, we next wanted to examine whether the Spo11 pre-mRNA splicing is changed in hnRNPH1 cKO spermatocytes. Although changed alternative splicing of Spo11 was not identified in our RNA-Seq data, considering the imperfect accuracy of RNA-seq, we decided to re-examine Spo11 splicing in P14 and P18 testes, isolated spermatocytes and spermatids from adult testis by RT-PCR, respectively.

As previously reported ${ }^{33}$, Spo11 showed a significantly changed alternative splicing $(S p o 11 \mathrm{a} / \beta)$ from P14 to P18, and the inclusion of its exon 2 is suppressed at the latter stage (Fig. $5 \mathrm{i}-\mathrm{j}$ ). However, upon hnRNPH1 ablation, this inhibition was relieved, and the expression of exon-2 of Spo11 maintained a high level at P18 (Fig. 5i-j). Interestingly, this change also existed in isolated spermatocytes but not round spermatids. Therefore, these results further indicate that hnRNPH1 could indeed regulate the splicing of Spo11 in spermatocytes, and hnRNPH1-deficiency in male germ cells leads to alternative splicing of Spo11 was compromised in the late meiosis, which may be one of the causes of sex-chromosome asynapsis.

\section{hnRNPH1 deletion in male germ cells impairs the communications between spermatogenic cells and Sertoli cells}

Because a large number of prematurely sloughed round spermatids were observed in hnRNPH1 cKO epididymis (Fig. 3j), we speculated that the attachment and communication between germ cells and Sertoli cells were destroyed before spermiation at stage VIII of spermatogenesis. To test this possibility, 
we first detected the RNA splicing of several cell adhesion- and junction-related genes identified from our RNA-Seq data of round spermatids by RT-PCR. Consequently, 8 out of 14 tested genes showed significant RNA alternative splicing changes in hnRNPH1 cKO round spermatids (Fig. 6a). WB result further confirmed that most alternative splicing-changed genes were also abnormally expressed in protein levels in hnRNPH1 cKO testes (Fig. 6b). Of note, the expression of TCF7L1/2 (two members of TCF family proteins) and IQSEC1 were enormously increased in hnRNPH1 cKO testes, especially in round spermatids (Fig. 6b-d). Based on the literature reports, all of these RNA alternative splicing changed-genes were important regulators for $\mathrm{Wnt} / \beta$-catenin activity ${ }^{34,35}$. While the specific role of these genes in spermatogenesis has not been elucidated, it should be noted that Wnt/ß-catenin was a critical cellular signal pathway that is crucial for multiple developmental stages during spermatogenesis ${ }^{36}$. More importantly, we found that $\beta$-catenin, known as a component of the cell-cell adhesion apparatus that has been demonstrated to regulate male germ cell differentiation ${ }^{37,38}$, displayed an abnormal distribution in hnRNPH1 cKO testes compared with control testes in which $\beta$-catenin was mainly localized at the bloodtestis barrier (BTB) (Fig. 6e).

In addition, given that F-ACTIN regulatory factors were thought to have critical roles in maintaining the adhesion of germ cells with Sertoli cells during germ cell development and movement towards the lumen ${ }^{39}$, we next tested the distribution of F-ACTIN in the hnRNPH1 cKO testes. In control testes, F-ACTIN usually appears in the region where Sertoli cells connect with spermatocytes or elongated spermatids (Fig. 6f). However, whether in adult (P56) or P24 hnRNPH1 cKO testes, F-ACTIN distributed in seminiferous tubules disorderly and abnormally located at the junction between Sertoli cells and round spermatids (Fig. 6f), which is akin to the phenotype observed in Ptbp2 cKO testes ${ }^{19}$. Furthermore, among the genes identified with alternative splicing and protein level-changes, Rapgef 6 and Fndc3a have been reported to mediate Sertoli cell-spermatid adhesion during mouse spermatogenesis, and both Rapgef6 and Fndc3a knockout mice showed male sterility ${ }^{40,41}$. Together, these results indicate that abnormal alternative splicing of the genes related to cell adhesion in hnRNPH 1 cKO spermatids leads to impairment of communication and junction between Sertoli cells and germ cells, thereby causing the phenotype of prematurely sloughed round spermatids in hnRNPH1 cKO testes.

\section{hnRNPH1 is essential for oogenesis and female fertility}

Considering that the Stra8-GFPCre used in this study can effectively induce deletion of the target gene in both male and female germlines, we thus aimed to define the physiological function of hnRNPH1 in oogenesis and female fertility. Inspiringly, after 5-months of fertility test, all hnRNPH1 cKO female mice were also showing complete sterile. We further performed HE stainings on the adult and P21 ovaries and found that the size of hnRNPH1 cKO ovaries was significantly smaller than controls, and almost no follicles were observed in hnRNPH1 cKO ovaries (Supplementary Fig.S8a). These results suggest a crucial role of hnRNPH1 in oogenesis and folliculogenesis. Interestingly, PTBP2 and SRSF3 were also found to be highly expressed in early oocytes, although their expression and localization were not affected in hnRNPH1 cKO ovaries (Supplementary Fig.S8b). 
To explore the molecular reasons of infertility in hnRNPH1 cKO females, we examined the expression of hnRNPH1 in the ovaries at embryonic day 17.5 (E17.5), when most female germ cells have developed to the pachytene stage. IF results showed that hnRNPH1 was strongly expressed in oocytes and granulosa cells of control mice but specifically absent in the oocytes of hnRNPH1 cKO mice (Fig. 7a), indicating that hnRNPH1 was successfully and specifically knocked out in oocytes. Since hnRNPH1 was localized in the meiotic stage of early oocytes and exhibited a similar expression pattern with in spermatocytes ( Supplementary Fig.S1f-g), we asked whether hnRNPH1 functions in the meiosis of early oocytes. To this aim, we detected the oocyte development efficiency in the ovaries at P1 and P3 by immunochemistry using a DDX4 antibody (a germ cell marker). Compared with control, the number of oocytes in the hnRNPH1 cKO ovary at P1 significantly reduced, and only a very few oocytes exist at P3 ovary (Fig. 7b). In addition, the TUNEL assay further revealed a substantially higher proportion of apoptotic oocytes in the hnRNPH1 cKO ovary at PO (Supplementary Fig.S8c). These results indicate that hnRNPH1 is essential for the early development of oocytes and plays a critical function in the meiosis of early oocytes.

To further determine the sub-stage of meiotic defects that occur in hnRNPH1 cKO oocytes, we first examined the meiotic processes of oocytes by chromosome spreading analyses at E17.5 ovaries. Similar to hnRNPH1 cKO spermatocytes, abnormal $\mathrm{YH} 2 \mathrm{AX}$ signal appeared in some hnRNPH1 cKO oocytes at the pachytene stage (Fig. 7c). Moreover, a higher percentage of serious asynapsis was observed in hnRNPH1 cKO oocytes than control oocytes based on the co-localization of SYCP1 and SYCP3 (Fig. 7d). Given the similar spatiotemporal expression and defective meiosis phenotype between spermatocytes and oocytes, we next asked whether hnRNPH1 regulates meiosis in oocytes by regulating RNA alternative splicing as in spermatocytes. As expected, we identified that two genes, Brme1 and Cpeb1, that have been reported to be involved in oogenesis and spermatogenesis ${ }^{42-44}$, exhibited abnormal alternative splicing in hnRNPH1 cKO ovaries (Fig. 7e). Inspiringly, some cell junction-related genes with aberrant alternative splicing in hnRNPH1 cKO testes, such as $T c f 7 / 1, T c f 7 / 2$, and $T c f 3$, were abnormally spliced in hnRNPH1 cKO ovaries as well (Fig. 7e). Likewise, TCF7L1/2 and their regulated protein $\beta$-catenin were also significantly increased in hnRNPH1 cKO oocytes (Fig. 7f-h). In addition, since hnRNPH1 can directly bind to Tcf3 and control its splicing to regulate the expression of E-cadherin ${ }^{45}$, another critical component of cell junction, we examined the E-cadherin expression in E17.5 ovaries. As a result, a significantly reduced expression of E-cadherin was observed in hnRNPH1 cKO ovaries (Fig. 7i). Taken together, in combination with the previous finding that aberrant distribution of $\beta$-catenin and/or E-cadherin could cause the globally prenatal oocyte attrition ${ }^{46,47}$, our data indicate that the abnormal splicing of genes functionally related to cell junction in hnRNPH1 cKO oocytes is supposed to be driving the early oocyte development and meiosis failure in hnRNPH1 cKO female mice.

\section{hnRNPH1 regulates the mRNA alternative splicing via recruiting splicing factors PTBP2 and SRSF3}

To decipher the underlying molecular mechanisms of how hnRNPH1 regulates the RNA alternative splicing of its target gene, we, therefore, performed RIP-sequencing (RIP-seq) for hnRNPH1 using purified WT germ cells from P28 testes. Annotation of the RIP-Seq data identified a total of 3,817 transcripts that 
were significantly enriched in the hnRNPH1 immunoprecipitants (cutoff: fold change $>2, P$-value $<0.05$ ) (Supplementary Table 7), and most of the hnRNPH1 binding sites ( 73.7\%) resided in the introns of genes encoding proteins (Fig. 8a). RNA-binding motif analysis indicated that hnRNPH1 preferred G-rich tracts of RNA (Fig. 8b), which is consistent with previous studies of hnRNPH1-RNA interactions ${ }^{48}$. Upon comparing RNA-seq and RIP-seq data, we found that 23\% (200 out of 881) and 22\% (205 out of 967) alternative splicing changed genes were bound by hnRNPH1 in spermatocytes and spermatids, respectively (Fig. 8c). Notably, GO enrichment analyses revealed that these alternative splicing changed genes bound by hnRNPH1 mainly were related to RNA splicing regulation in both spermatocytes and spermatids (Supplementary Fig.S9a-b), indicating an essential role of hnRNPH1 in regulating many splicing factors, which may involve more other splicing events. Moreover, a large number of differentiallyexpressed transcripts were also found to be bound by hnRNPH1 (Supplementary Fig.S9c), suggesting a splicing-independent mechanism underling hnRNPH1 regulates gene expression.

Since PTPB2 has a similar role in RNA alternative splicing regulation in germ cells, we next explored whether PTBP2 could bind hnRNPH1 target transcripts by comparing the reported CLIP-seq data of PTPB $2^{19}$ with our RIP-seq data. The results showed that many alternative splicing changed genes were co-bound by hnRNPH1 and PTBP2 in spermatocytes and spermatids (Supplementary Fig.S9d). More interestingly, these genes were closely related to RNA splicing, meiotic nuclear division, and cell junction (Supplementary Fig.S9e-f). We next examined whether the alternative splicing-changed RNA splicing regulated genes were abnormal in splicing level because most of the alternative splicing-changed genes that appeared in hnRNPH1 cKO germ cells were not directly bound by hnRNPH1 (Fig. 8c) but likely resulted from alterations in the splicing factors and their respective downstream targets. In comparison with control, among 8 examined genes, Hnrnpa2b1, Hnrnph3, Clk1, and Rbm5 showed significant splicing changes in hnRNPH1 cKO germ cells (Supplementary Fig.S10a). Interestingly, we found three RBM5 target genes, Anks3, Rangap1, and $\mathrm{Cftr}^{17}$, also exhibited abnormal alternative splicing in hnRNPH1 cKO germ cells (Supplementary Fig.S10b). We next performed RIP-qPCR and confirmed that these three genes were bound by RBM5 but not hnRNPH1 (Supplementary Fig. S10c-d), indicating that some genes with abnormal alternative splicing events in hnRNPH1 cKO germ cells are not directly regulated by hnRNPH1 but by its target genes, especially the splicing factors.

Considering the ability of hnRNPH1 to interact with splicing factors PTBP2 and SRSF3 in testes, we further explored whether the alternative splicing changed genes in hnRNPH1 cKO germ cells were bound and regulated by PTBP2 and SRSF3. Thus, we performed RIP-qPCR using the anti-hnRNPH1, PTBP2, and SRSF3 antibodies to immunoprecipitate the pre-mRNAs from purified germ cells, respectively. We chose 11 alternative splicing changed genes here were included in our RIP-seq data for further qPCR assay. The results showed that 6 out of selected genes (Spo11, Cpeb1, Rapgef6, Rbm5, Hnrnpa2b1, and Clk1) were simultaneously enriched in hnRNPH1, PTBP2, and SRSF3 immunoprecipitants (Fig. 8d). Next, we performed the overexpression and knockdown experiments in HEK293T cells in vitro to investigate whether PTBP2 and SRSF3 could regulate the above six genes in splicing level. Interestingly, we found that the alternative splicing of four genes (Spo11, Cpeb1, Rapgef6, and Rbm5) was changed in the same 
direction when hnRNPH1, PTBP2, and SRSF3 were either overexpressed or knocked down, respectively (Fig. 8e and Supplementary Fig.S11a). Significant co-localization of hnRNPH1 and PTBP2 at the indicated exons of the four target genes was confirmed by the RIP-seq and CLIP-seq experiments (Fig. 8f). These results revealed that hnRNPH1 target genes could be regulated by PTBP2 and SRSF3 in splicing level.We further examined the binding affinity of the four target genes to PTBP2 and SRSF3 by RIP-qPCR assay in control and hnRNPH1 cKO germ cells. Unexpectedly, the enrichment level of all the four hnRNPH1 target genes bound to PTBP2 and SRSF3 protein significantly decreased in hnRNPH1 cKO germ cells compared with that in control (Fig. 9a-b). A similar phenomenon was observed when hnRNPH1 was knocked down in HEK293T cells in vitro (Supplementary Fig.S11b-c). Taken together, our data suggest that hnRNPH1 could recruit PTBP2 and SRSF3 to the corresponding positions of target genes and regulate their mRNA splicing.

\section{Discussion}

Spermatogenesis is a complex process dependent on the coordinated regulation of gene expression at transcriptional and post-transcriptional levels ${ }^{49}, 50$. Many RNA-binding proteins (RBPs), especially some critical splicing factors, have been involved in regulating spermatogenesis and played a role in different types of spermatogenic cells to ensure the orderly differentiation of spermatogenic cells because each spermatogenic developmental stage has its own unique alternative splicing program ${ }^{51}$. For instance, BCAS2 could regulate meiosis initiation by promoting appropriate pre-mRNA splicing in undifferentiated spermatogonia ${ }^{52}$, while MRG15, which was highly expressed at the onset of round spermatid differentiation, contributes to spermiogenesis by participating in mRNA splicing ${ }^{15}$. In the current study, we found that hnRNPH1 is mainly expressed in spermatocytes and round spermatids and involved in the alternative splicing regulation during the meiotic and post-meiotic stages. Increasing evidence indicated that many RBPs have direct roles in transcription, as exemplified by the elucidated function of particular splicing regulators in transcription, including SRSF2 ${ }^{53}, \mathrm{HNRNPK}^{54}, \mathrm{HNRNPL}^{55}$, and RBFOX2 ${ }^{56}$. Like transcription factors (TFs), RBPs also showed an obvious preference for hotspots in the genome, particularly gene promoters frequently associated with transcriptional output ${ }^{57}$.

It is noteworthy that among the hnRNPH1-interacting proteins identified in our IP-MS data, some TFs related to gene silencings, such as Cirbp and Smarca5, have been reported to be involved in spermatogenesis ${ }^{58}$ and oogenesis ${ }^{59}$. Interestingly, most of the differentially expressed genes caused by hnRNPH1 deletion were up-regulated; thus, we speculated that hnRNPH1 might cooperate with specific TFs to mainly silence gene transcription in spermatogenic cells. Herein, our whole transcriptome profiling of spermatocytes and spermatids revealed a comprehensive splicing program that is susceptible to ablation of hnRNPH1 and affects genes of essential relevance for spermatogenesis. Indeed, a large number of splicing events normally occurred during the transition from spermatocytes to post-meiotic germ cells ${ }^{14}$, some of which may be meditated by hnRNPH1 because of a spatiotemporal decreasing trend of hnRNPH1 expression from spermatocytes to round spermatids was observed in this study. However, it is important to note that our RNA-seq data showed that hnRNPH1 ablation leads to 
dysregulation in the mRNA expression of a large number of genes, but most are likely not caused by abnormal alternative splicing, suggesting an independent role of hnRNPH1 in transcriptional regulation during spermatogenesis.

As an RNA binding protein, hnRNPH1 can directly bind to the mRNAs of some target genes for regulation. Indeed, our RIP-seq data indicated that hnRNPH1 indeed directly binds more than 3800 genes; however, only a small number of genes with differential expression or splicing-changes identified from hnRNPH1 cKO spermatogenic cells could binding to hnRNPH1. Of note, in the current study, hnRNPH1 was identified to bind and regulate the alternative splicing of genes encoding splicing factors (e.g., $R b m 5$, Hnrnpa2b1, Clk1, and $h n R N P H 3)$, suggesting hnRNPH1 may regulate the gene alternative splicing by cooperatively modulating some splicing factors not directly regulate its target genes' splicing. It is reasonable to infer that dysregulation of these splicing factors inevitably would lead to more splicing errors in their target genes, thus amplifying the initial adverse effects and generating a vicious circle of aberrant splicing. As previously reported, hnRNPH1 is supposed to be one of the core components of the splicing machinery regulating the dynamic balance of mRNA splicing, and in this study, it was found to play an equally critical role in spermatogenesis. In addition, while hnRNPH1 mostly binds intronic sequences, binding sites in coding and non-coding exonic regions were also observed in this study (Fig. 8a), raising the possibility that hnRNPH1 may have splicing-independent roles in post-transcriptional regulation in germ cells. Many potential interacting proteins are related to translation were identified from the immunoprecipitants of hnRNPH1, which further supported this possibility. Moreover, a previous study has also clarified the role of hnRNPH1 in splicing, mRNA decay, and translation through a suite of highthroughput approaches ${ }^{48}$. Therefore, in addition to modulating alternative splicing, we cannot exclude the possible functions of hnRNPH1 on the post-transcriptional regulation during spermatogenesis. Of note, two key splicing factors, PTBP2 and SRSF3, were identified to interplay with hnRNPH1 in spermatogenic cells in this study, and many genes with identical splicing events regulated by hnRNPH1 and PTBP2 were found to be involved in several crucial signaling pathways related to reproduction. However, a number of splicing defects found in the hnRNPH1 cKO germ cells were not altered in the PTBP2 null germ cell, suggesting that hnRNPH1 and PTBP2 share some but not all splicing events. Another key splicing factor, SRSF3, has been reported to be bound by DDX5 to regulate splicing in spermatogonia ${ }^{29}$ and was identified to regulate the splicing of hnRNPH1 target genes in the current study. More interestingly, the binding affinity of PTBP2 and SRSF3 to hnRNPH1 target genes (e.g., Spo11, Cpeb1, Tcf7l2, and Rbm5) was significantly reduced when hnRNPH1 was ablated (Fig. 9a-b), indicating that PTBP2 and SRSF3 could recruit the hnRNPH1 target genes to regulate its alternative splicing events. Nevertheless, PTBP2 and SRSF3 were only recruited to a small subset of hnRNPH1 target genes; the molecular mechanisms mediating the selective association of PTBP2 and SRSF3 with hnRNPH1 at specific target genes need to be elucidated in the future.

Noteworthy, many genes showing abnormal alternative splicing identified in hnRNPH1 cKO spermatocytes were essential for the completion of meiosis. For instance, Ehmt2 ${ }^{60}$, Spo $16^{61}$, Suv39h ${ }^{62}$, and $B r m e 1^{63}$ have been reported to be involved in multiple meiotic progressions such as synapsis, 
recombination, and crossover formation. In this study, most of these genes that were verified to be splicing-changed have abnormal protein expression, which was the possible reason why some spermatocytes showed incomplete synapsis and DSB repair. Moreover, a group of hnRNPH1 cKO spermatocytes that appeared unsynapsis between $\mathrm{X}$ - and $\mathrm{Y}$-chromosome was likely due to lack of proper Spo11 a subtype because aberrant Spo11 splicing could give rise to a higher risk of sex chromosomes aneuploidy ${ }^{6}$. Another interesting finding in the current study is that the female hnRNPH 1 cKO mice were also infertile and showed similar meiotic defects with hnRNPH1 cKO male mice. Intriguingly, some of the meiosis-related genes (e.g., Brme1 and Cpeb1) also showed abnormal alternative splicing in the hnRNPH1 cKO ovaries, and the mutant mice of these genes exhibited both male and female sterility as well ${ }^{44,64}$. Thus, these abnormal splicing events in meiotic genes resulting from hnRNPH1 ablation may be responsible for losing spermatocytes and meiosis delay.

During spermatogenesis, male germ cells must maintain stable attachment with Sertoli cells via testisunique intermediate filament- and actin-based cell junctions to prevent sloughing of immature germ cells from the seminiferous epithelium ${ }^{39}$. In this study, a large number of prematurely sloughed round spermatids were observed in the epididymis of hnRNPH1 cKO testes, suggesting that hnRNPH1 may regulate the cell junction and adhesion between spermatogenic cells and Sertoli cells. Similarly, in the Ptbp2 mutant mice ${ }^{16}$, spermatogenic cells showed increased apoptosis and premature release into the lumen, as observed in the hnRNPH1 cKO mice. Additionally, many genes functionally related to cell adhesion exhibited abnormal alternative splicing upon hnRNPH1 ablation in spermatogenic cells (especially in round spermatids), further supporting the early spermatids released phenotype in hnRNPH1 cKO testes. Interestingly, whether in spermatocytes or round spermatids, many abnormal splicing events caused by hnRNPH1 ablation were consistent with that in Ptbp2 null testes. However, it is worth noting that many genes such as $T c f 7 / 1$ and $T c f 7 / 2$ were significantly up-regulated in hnRNPH1 $c K O$ spermatogenic cells, but it was likely further induced by the overexpression of $\beta$-catenin ${ }^{34}$. As a matter of fact, the germ cells sloughing from the seminiferous tubules accompanied by an overall increase of $\beta$ catenin in the epithelium resembled a common manifestation of disordered BTB structure. However, hnRNPH1, as a transcriptional or post-transcriptional regulatory factor, is unlikely to directly participate in the dynamic regulatory process of BTB opening and closing. Actin regulatory factors were thought to have critical roles in maintaining the adhesion of Sertoli cells with germ cells during their development and movement towards the lumen. Since most of the abnormally expressed proteins related to cell adhesion could regulate F-actin remodeling ${ }^{65}$, it is plausible to observe disordered F-actin distribution in hnRNPH1 cKO testes similar to the features of Ptbp2 cKO testes showing a defect in germ-Sertoli cell communication attributed to the loss of Sertoli cell polarity. Another more critical evidence for this phenotype is that among these abnormally spliced genes, Rapgef $6^{40}$, Fndc $3 a^{41}$, and $R b m 5^{66}$ have been reported to mediate spermatid-Sertoli adhesion during mouse spermatogenesis, and their expression was significantly changed in hnRNPH1 cKO germ cells due to abnormal splicing, which may also contribute to the disorder of cell-cell junction and communication caused by hnRNPH1 ablation. 
Furthermore, although the abnormality of the first meiosis also occurred in the hnRNPH1 cKO female germ cells, the phenotype seems to be much more severe than that in the male germ cells because the early oocytes almost disappeared at P3. In fact, in hnRNPH1 cKO ovaries, the proportion of oocytes with abnormal localization of $\mathrm{YH} 2 \mathrm{AX}$ or SYCP1 was only approximately $20 \%$; thus, the defect of DSB repair or synapsis should not to be the only cause of oogenesis arrest at an early stage. Surprisingly, many cell junction-related genes with aberrant alternative splicing in hnRNPH1 cKO testes, such as $T c f 7 / 1, T c f 7 / 2$, and Tcf3, were also abnormally spliced in hnRNPH1 $\mathrm{cKO}$ ovaries, resulting in dysregulation of their target genes encoding $\beta$-catenin and E-cadherin, which have important roles in maintenance between oocytes and granulosa cells. Considering that aberrant distribution of $\beta$-catenin or E-cadherin can cause the globally prenatal oocyte attrition, the abnormal splicing of genes functionally related to cell junction was another critical reason for early oocyte loss in female hnRNPH1 cKO mice.

In summary, our study uncovers an essential role of hnRNPH1 in recruiting key splicing factors such as PTBP2 and SRSF3 to coordinate proper alternative splicing of its target mRNA transcripts, and establishes a model whereby hnRNPH1 controls a network of alternative splicing events in germ cells necessary to establish the correct protein isoforms required for meiosis and germ-Sertoli (or granulosa) cell crosstalk in mice (Fig. 9c). This research dramatically expands our understanding of alternative splicing machinery regulating spermatogenesis through close cooperation between splicing factors.

\section{Materials And Methods}

\section{Ethics statement}

All the experimental animal procedures were approved by the Institutional Animal Care and Use Committee of Tongji Medical College, Huazhong University of Science and Technology, and the mice were fed in specific pathogen-free facilities of Huazhong University of Science and Technology. All mouse experiments were conducted following the Guide for the Care and Use of Laboratory Animal guidelines.

\section{Mouse generation and genotyping}

Floxed hnRNPH1 mice (Cat\# T001430) were purchased from the Model Animal Research Center of Nanjing University. In brief, the mice were generated by embryonic stem cells (ESCs) targeting and blastocyst injection. ESCs were targeted by carrying two loxP sites flanked in exon 6 and a neomycin selection cassette flanked by FRT sites in intron 5-6 of the Hnrnph1 gene. The Hnrnph $1^{+/ \text {flox }}$ mice were obtained by chimera formation and germline transmission. Mice were then crossed with FLP transgenic mice to remove the neomycin cassette and maintained on a C57BL/6J background. Stra8-GFPCre mouse line in the C57BL/6J background was obtained from Dr. Minghan Tong's Laboratory at Center for Excellence in Molecular Cell Science, Chinese Academy of Sciences. Stra8-GFPCre males were first crossed with Hnrnph $7^{\text {flox/flox }}$ females to generate the Stra8-GFPCre; Hnrnph $7^{+/ \text {flox }}$ males, then the Stra8GFPCre, Hnrnph 1 $^{\text {/flox }}$ male mice were bred with Hnrnph $7^{\text {flox/flox }}$ female mice to obtain the Stra8-GFPCre, $H n r n p h 7^{\text {flox/ }} \triangle$ (designated as hnRNPH1 cKO) male and female mice. For the global Hnrnph1 knockout 
mouse generation, we bred $H n r n p h 7^{\text {flox } / \triangle}$ male mice with $H n r n p h 7^{\text {flox } / \triangle}$ female mice to generate Hnrnph $1^{\triangle / \triangle}$ mice (designated as hnRNPH1 gKO). Mouse genotyping was performed by PCR amplification of genomic DNA extracted from mouse tails. The PCR primer sequences are showed in Supplementary Table S9.

Fertility test

Fertility was tested in control and hnRNPH1 cKO mice after 8-weeks-old. Each hnRNPH1 cKO male or female mouse was caged with one WT C57BL/6J female or male mouse (8-12 weeks), respectively, and the females were checked for vaginal plugs every morning. The plugged females were separated and single caged, and the pregnancy was recorded. The fertility test lasted for at least five months.

\section{Histological analysis, immunostaining and imaging}

Mouse testes, epididymides, and ovaries were collected immediately after euthanasia. Samples were fixed in Bouin's solution (Sigma, HT10132) at $4^{\circ} \mathrm{C}$ overnight and then embedded in paraffin. $5 \mu \mathrm{m}$ sections were prepared and mounted on glass slides. Hematoxylin and eosin (H\&E) staining, periodic acid-Schiff (PAS) staining, and immunohistochemistry were performed using a standard protocol. For immunofluorescence staining, samples were fixed in $4 \%$ paraformaldehyde (PFA) in PBS overnight at $4^{\circ} \mathrm{C}$ and were dehydrated sequentially and then embedded in Tissue-Tek optimal cutting temperature (O.C.T) compound. Cryosections were prepared at $5 \mu \mathrm{m}$. For antigen retrieval, slides were boiled in $0.01 \mathrm{M}$ sodium citrate buffer ( $\mathrm{pH}=6.0$ ) for 20 min using a microwave. After a brief wash with 1x PBS, sections were blocked using blocking solution (containing 3\% normal goat serum and $3 \%$ fetal bovine serum in $1 \%$ bovine serum albumin) for $1 \mathrm{~h}$ at room temperature (RT). The sections were incubated with primary antibodies at $4^{\circ} \mathrm{C}$ for overnight. After washing with PBS, Alexa Fluor 488 goat anti-rabbit IgG (1:500; A32731, Invitrogen) and/or Alexa Fluor 594 goat anti-mouse lgG (1:500, A11032, Invitrogen) secondary antibodies were added and incubated for $1.5 \mathrm{~h}$ at RT, after washing with PBS and mounted using Vectorshield mounting media with DAPI (H1200, Vector laboratories). Laser confocal scanning images were captured using FluoView 1000 microscope (Olympus, Japan) with a digital camera (MSX2, Microshot Technology Limited, China). The details of primary antibodies used in this study are showed in Supplementary Table S8.

\section{TUNEL analyses}

TUNEL assay was performed with One Step TUNEL Apoptosis Assay Kit (Meilunbio, MA0223) following the manufacturer's instructions using testes and ovaries cryosections.

\section{Meiotic chromosome spread analyses}

Chromosomes spreads were prepared as previously reported with slight modifications ${ }^{67}$. In brief, testicular seminiferous tubules were pretreated by hypotonic buffer ( $30 \mathrm{mM}$ Tris, $50 \mathrm{mM}$ sucrose, $17 \mathrm{mM}$ trisodium citrate dihydrate, $5 \mathrm{mM}$ EDTA, $0.5 \mathrm{mM}$ DTT and $0.5 \mathrm{mM}$ phenylmethylsulphonyl fluoride (PMSF), $\mathrm{pH}=8.2$ ) for $60 \mathrm{~min}$. The tubules were then cut for several short fragments and were suspended in $100 \mathrm{M}$ sucrose for dispersing to single cells and spreading to a thin cell layer on slides. Followed with 
$2 \%(\mathrm{w} / \mathrm{v})$ paraformaldehyde solution containing $0.15 \%$ Triton X-100 treatment, the prepared slides were placed in a humidified chamber for $4 \mathrm{~h}$ at RT and washed with $0.4 \%$ Photo-Flo (Kodak, 1464510). The slides were then air-dried at RT for 15-30 min and then blocked with blocking solution (containing 5\% normal donkey serum) for $1 \mathrm{~h}$ for immunostaining.

\section{Plasmids and minigenes costruction}

Full-length and indicated fragments of Hnrnph1 cDNA was cloned into a pCMV vector containing the Nterminal Flag epitope tag, and full-length and indicated fragments of Srsf3 and Ptbp2 cDNAs were cloned into a pCMV vector containing the N-terminal c-Myc epitope tag. HEK293T cells were transfected with indicated plasmids using Lipofectamine 2000 (Life Technologies). After 36 h, immunoprecipitation was performed using anti-Flag rabbit polyclonal antibody ( $5 \mu \mathrm{g}$ antibody in $500 \mu \mathrm{l}$ cell lysate, Cat\#: 20543-1AP, Proteintech), followed by a western blot to identify protein interactions. For minigenes construction, the Spo11, Cpeb1, Tcf712, Rbm5, Anks3, Rangap1, Cftr, and Tcf3 minigenes were amplified from genomic DNA of adult mouse testis using primers shown in Supplementary Table S9, and then the minigenes were cloned into pCDNA3.1(-) vector and validated by sequencing.

\section{Cell culture and transfection}

HEK293T cells were cultured in the DMEM medium with 10\% fetal bovine serum (Gibco, 10270106). Transfection was performed with Lipofectamine 2000 (Invitrogen) according to the manufacturer's instructions. For RNA interference, cells were transfected with appropriate siRNAs (Sigma-Aldrich) using Lipofectamine RNAiMAX (Invitrogen) and harvested $48 \mathrm{~h}$ later for analyses.

\section{Purification of spermatogenic cells}

The purification of spermatogenic cells was performed as described previously ${ }^{68}$. The STA-PUT method was used based on sedimentation velocity at unit gravity to purify the pachytene spermatocytes and round spermatids from adult control and hnRNPH1 $\mathrm{cKO}$ mouse testes. The isolated spermatogenic cells were further confirmed by their distinct nuclear morphology (DAPI staining of nuclei). Pachytene spermatocytes and round spermatids with $\geq 90 \%$ purity were used for RNA-seq analyses.

\section{RT-PCR and qPCR}

RNAs were extracted from indicated tissues using TRIzol reagent (Invitrogen), digested with Rnase-free DNase (Roche), and 1 ug of RNA was reverse-transcribed into cDNAs using the PrimeScript RT Reagent Kit (Takara) according to the manufacturer's protocol. RT-PCR primers were designed to amplify two or multiple isoforms with different sizes, are showed in Supplementary Table S9. PCR products were quantified using Image J software. Splicing ratios were represented as PSI (Percent Spliced In) value, representing the percentage of a gene's mRNA transcripts that included a specific exon or splice site. qPCR was performed with SYBR green master mix (TaKaRa) on LightCycler@96 Real-Time PCR system (Roche) according to manufacture's instructions.

\section{Western blotting}


The indicated samples were collected, and proteins were extracted by using RIPA buffer (CWBIO, Cat\# 01408). Protein extracts were denatured with $5 \times$ SDS loading buffer (Beytime, P0015L) at $100^{\circ} \mathrm{C}$ for 10 min and run on a $10 \%$ SDS-PAGE, then transferred to PVDF membrane (Bio-Rad). After being blocked in $5 \%$ skimmed milk at RT for $1 \mathrm{~h}$, the primary antibodies were incubated overnight at $4^{\circ} \mathrm{C}$. After washing, the membrane was incubated with the secondary antibody for $1 \mathrm{~h}$ at RT, and then photographed using the Luminol/enhancer solution and Peroxide solution (ClarityTM Western ECL Substrate, Bio-Rad). The details of primary antibodies and secondary antibodies used in this study are showed in Supplementary Table S8.

\section{Immunoprecipitation}

Mouse tissues or cells were homogenized in cell lysis buffer, and the resultant cell extracts were treated with or without RNase $A(1 \mu \mathrm{g} / \mathrm{ml})$ at $4^{\circ} \mathrm{C}$ for $1 \mathrm{~h}$, followed by the lysates were clarified by centrifugation at $12,000 \times \mathrm{g}$. Then the relevant antibodies and pre-cleaned magnetic protein $\mathrm{A} / \mathrm{G}$ beads were incubated with the tissue lysates at $4^{\circ} \mathrm{C}$ for overnight. The beads were washed with Cell lysis buffer for Western and IP (Beytime, P0013) with protease inhibitor cocktail (P1010, Beyotime) and then boiled in 5× SDS loading buffer (Beytime, P0015L) for western blotting analyses.

\section{RNA immunoprecipitation, RIP-Seq, RNA-Seq and bioinformatics analyses}

RNA immunoprecipitation (RIP) was performed using testes of 4-weeks-old mice. After the testes were dissected and decapsulated in $1 \times$ PBS buffer at RT, the seminiferous tubules were lysed in buffer containing $100 \mathrm{mM} \mathrm{KCl}, 10 \mathrm{mM}$ HEPES ( $\mathrm{pH}=7.0$ ), 0.5\% Triton X-100, $5 \mathrm{mM} \mathrm{MgCl}, 1 \mathrm{mM} \mathrm{DTT}, 0.5 \%$ NP40, RNase inhibitor (100 U/ml) (Invitrogen), and EDTA-free proteinase inhibitor (Roche). Then, the testicular lysate was passed through a 27.5 gauge needle 5 times to promote nuclear lysis, followed by brief sonication using a Bioruptor 200 (Diagenode). After incubation on ice for 20 min, the nuclear lysate was added with $50 \mathrm{ul}$ beads for pre-cleared incubation at $4^{\circ} \mathrm{C}$ for $1 \mathrm{~h}$. For each reaction, $5 \mu \mathrm{g} \mathrm{hnRNPH} 1$ antibody (or lgG for controls) was incubated with protein G-Dyna beads in $1 \mathrm{ml}$ NT2 buffer. After shaking at $4^{\circ} \mathrm{C}$ for $4 \mathrm{hrs}$, control IgG and antibody-coated beads were incubated with testicular extracts and kept shaking gently overnight at $4^{\circ} \mathrm{C}$. The next day, the bead complexes containing antibodies, target proteins, and RNA were washed for $20 \mathrm{~min}$ at $4^{\circ} \mathrm{C}$ and repeated 3 times. Protein-bound mRNAs were then extracted using an RNA extraction kit (ZYMO research). The purity and integrity of eluted RNA were assessed using an Agilent Bioanalyzer. 1-2 $\mu \mathrm{g}$ of cDNAs were synthesized from the extracted RNA using the Clontech SMARTer cDNA kit (Clontech Laboratories, CA USA, catalog\# 634938), and adaptors were removed through digestion with Rsal. According to the manufacturer's protocol, the cDNAs were fragmented using an ultrasonicator and profiled using an Agilent Bioanalyzer, followed by subjecting to Illumina library preparation using NEBNext reagents (New England Biolabs, Ipswich, MA USA, catalog\# E6040). Then the size distribution, quantity and quality of the Illumina libraries were measured by the Agilent Bioanalyzer. The libraries were submitted for Illumina HiSeq2000 sequencing (Otogenetics, Norcross, GA). Paired-end 100 nucleotide (nt) reads were generated, and the data quality was checked using FASTQC (Babraham 
Institute, Cambridge, UK). The raw data were then subjected to data analysis using Tophat2 and Cufflinks. Two biological replicates were analyzed for each sample.

For RNA-Seq, total RNA was extracted from pachytene spermatocytes and round spermatids that were sorted from adult control and hnRNPH1 cKO testes using TRIzol reagents (Invitrogen) following the manufacturer's protocol with three biological repeats, respectively. The concentrations and integrity of RNA were verified using a NanoDrop 2000 Spectrophotometer (Thermo Fisher Scientific). Then, a total amount of $2 \mu \mathrm{g}$ of RNA for each sample was used to prepare poly(A+)-enriched cDNA libraries using the NEBNext Ultra RNA Library Prep Kit for Illumina (New England Biolabs), and raw data (base pairs) were generated by the Illumina Hi-Seq 2500 platform. Raw reads were processed with cutadapt v1.9.1 to remove adaptors and perform quality trimming, and trimmed reads were mapped to the UCSC mm10 assembly using HiSAT2 (V2.0.1) with default parameters. Differentially expressed genes for all pairwise comparisons were measured by DESeq2 (v1.10.1) with internal normalization of reads to account for library size and RNA composition bias. Differentially regulated genes in the DESeq2 analysis were defined as 2 -fold changes with an adjusted $P$-value of $<0.05$. Gene Ontology (GO) and KEGG analyses were performed using the database for DAVID. rMATS was used to analyze the alternative splicing events between control and hnRNPH1 cKO groups. To detect valid alternative splicing events, those with false discovery rate $(F D R)<0.05$ and $|\triangle P S| \mid>10 \%$ were categorized as differential alternative splicing events, which were classified into five types, including the retained intron (RI), skipped exon (SE), alternative 5' splice site (A5SS), Mutually exclusive exons (MXE), alternative 3' splice site (A3SS). The rmats2sashimiplot was used to convert the rMATS output into Sashimi plot.

\section{Statistical analyses}

The data are shown as the mean \pm standard deviation (SD). GraphPad Prism 7.0 software (GraphPad, San Diego, CA, USA) was used for the statistical analyses. Significant differences between the two groups were analyzed using the Student's $t$-test, and that between multiple groups was measured using one-way analysis of variance followed by Bonferroni post hoc tests. A value of $P<0.05$ was considered statistically significant.

\section{Declarations}

\section{Acknowledgments}

We would like to thank Dr. Minghan Tong at Shanghai Institute of Biochemistry and Cell Biology, Chinese Academy of Sciences, for kindly sharing the Stra8-GFPCre mouse line.

\section{Funding}

This work, in part, was supported by grants from the National Natural Science Foundation of China (81971444 to S.Y., 31900606 to S.F., and 31801237 to X.W.) and the Strategic Collaborative Research 
Program of the Ferring Institute of Reproductive Medicine, Ferring Pharmaceuticals and Chinese Academy of Sciences (FIRMSC200502 COV02 to S.Y.).

\section{Author contributions}

S.F. and S.Y. conceived and designed the study. S.F., J.L., H.W., K.L., Y.G., Y.W., and X.W. performed all bench experiments and data analyses. S.F. and S.Y. wrote the manuscript. S.Y. supervised the project. All authors read and approved the manuscript.

\section{Competing interests}

The authors declare that they have no competing interests.

\section{Data and materials availability}

All data needed to evaluate the conclusions in the paper are present in the article and/or the Supplementary Materials. All RNA-seq and RIP-seq data are deposited in the NCBI SRA (Sequence Read Achieve) database (PRJNA771927).

\section{References}

1. Griswold, M.D. Spermatogenesis: The Commitment to Meiosis. Physiological reviews 96, 1-17 (2016).

2. Song, H., Wang, L., Chen, D. \& Li, F. The function of Pre-MRNA alternative splicing in mammal spermatogenesis. International journal of biological sciences 16, 38 (2020).

3. Nilsen, T.W. \& Graveley, B.R. Expansion of the eukaryotic proteome by alternative splicing. Nature 463, 457-463 (2010).

4. Bellani, M.A., Boateng, K.A., McLeod, D. \& Camerini-Otero, R.D. The expression profile of the major mouse SP011 isoforms indicates that SP011beta introduces double strand breaks and suggests that SP011alpha has an additional role in prophase in both spermatocytes and oocytes. Molecular and cellular biology 30, 4391-4403 (2010).

5. Chen, Y. et al. Single-cell RNA-seq uncovers dynamic processes and critical regulators in mouse spermatogenesis. Cell research 28, 879-896 (2018). 
6. Kauppi, L. et al. Distinct properties of the XY pseudoautosomal region crucial for male meiosis. Science 331, 916-920 (2011).

7. Sette, C., Dolci, S., Geremia, R. \& Rossi, P. The role of stem cell factor and of alternative c-kit gene products in the establishment, maintenance and function of germ cells. International Journal of Developmental Biology 44, 599-608 (2004).

8. Li, L. et al. Molecular cloning and characterization of a novel transcript variant of Mtsarg1 gene. Molecular biology reports 36, 1023-1032 (2009).

9. Walker, W.H., Sanborn, B.M. \& Habener, J.F. An isoform of transcription factor CREM expressed during spermatogenesis lacks the phosphorylation domain and represses CAMP-induced transcription. Proceedings of the National Academy of Sciences 91, 12423-12427 (1994).

10. Kanemori, Y. et al. Two functional forms of ACRBP/sp32 are produced by pre-mRNA alternative splicing in the mouse. Biology of reproduction 88, 105 (2013).

11. Uchibe, K., Shimizu, H., Yokoyama, S., Kuboki, T. \& Asahara, H. Identification of novel transcription-regulating genes expressed during murine molar development. Developmental Dynamics 241, 1217-1226 (2012).

12. Kanai, Y. et al. Identification of two Sox17 messenger RNA isoforms, with and without the high mobility group box region, and their differential expression in mouse spermatogenesis. The Journal of cell biology 133, 667-681 (1996).

13. Wahl, M.C., Will, C.L. \& Lührmann, R. The spliceosome: design principles of a dynamic RNP machine. Cel/ 136, 701-718 (2009).

14. Naro, C. et al. Functional Interaction between U1snRNP and Sam68 insures proper 3' end premRNA processing during germ cell differentiation. Cell reports 26, 2929-2941. e2925 (2019).

15. Iwamori, N. et al. MRG15 is required for pre-mRNA splicing and spermatogenesis. Proceedings of the National Academy of Sciences 113, E5408-E5415 (2016).

16. Zagore, L.L. et al. RNA binding protein Ptbp2 is essential for male germ cell development. Molecular and cellular biology 35, 4030-4042 (2015).

17. O'Bryan, M.K. et al. RBM5 Is a Male Germ Cell Splicing Factor and Is Required for Spermatid Differentiation and Male Fertility. Plos Genetics 9 (2013).

18. Liu, W.B. et al. BCAS2 is involved in alternative mRNA splicing in spermatogonia and the transition to meiosis. Nature Communications 8 (2017). 
19. Hannigan, M.M., Zagore, L.L. \& Licatalosi, D.D. Ptbp2 Controls an Alternative Splicing Network Required for Cell Communication during Spermatogenesis. Cell Rep 19, 2598-2612 (2017).

20. Han, S.P., Tang, Y.H. \& Smith, R. Functional diversity of the hnRNPs: past, present and perspectives. Biochem J 430, 379-392 (2010).

21. Huelga, S.C. et al. Integrative Genome-wide Analysis Reveals Cooperative Regulation of Alternative Splicing by hnRNP Proteins. Cell Reports 1, 167-178 (2012).

22. $L i$, J. et al. HnRNPL as a key factor in spermatogenesis: Lesson from functional proteomic studies of azoospermia patients with sertoli cell only syndrome. J Proteomics 75, 2879-2891 (2012).

23. $\mathrm{Xu}, \mathrm{H}$. et al. Expression analysis of multifunctional RNA-binding protein hnRNP K during development of mammalian testis. Pol J Vet Sci 21, 343-351 (2018).

24. Liu, Y. et al. The germ cell nuclear proteins hnRNP GT and RBMY activate a testis-specific exon. PLoS genetics 5, e1000707 (2009).

25. Prudencio, M. et al. Distinct brain transcriptome profiles in C9orf72-associated and sporadic ALS. Nature neuroscience 18, 1175-1182 (2015).

26. Wu, Y. et al. HNRNPH1-stabilized LINC00662 promotes ovarian cancer progression by activating the GRP78/p38 pathway. Oncogene, 1-13 (2021).

27. Uren, P.J. et al. High-throughput analyses of hnRNP H1 dissects its multi-functional aspect. Rna Bio/ 13, 400-411 (2016).

28. Hannigan, M.M., Zagore, L.L. \& Licatalosi, D.D. Ptbp2 controls an alternative splicing network required for cell communication during spermatogenesis. Cell reports 19, 2598-2612 (2017).

29. Legrand, J.M. et al. DDX5 plays essential transcriptional and post-transcriptional roles in the maintenance and function of spermatogonia. Nature communications 10, 1-21 (2019).

30. Lin, Z. et al. Mett|3-/Mett|14-mediated mRNA N(6)-methyladenosine modulates murine spermatogenesis. Cell Res 27, 1216-1230 (2017).

31. Koubova, J. et al. Retinoic acid regulates sex-specific timing of meiotic initiation in mice. Proc Natl Acad Sci U S A 103, 2474-2479 (2006).

32. Kauppi, L. et al. Distinct properties of the XY pseudoautosomal region crucial for male meiosis. Science (New York, N.Y.) 331, 916-920 (2011).

33. Cesari, E. et al. Combinatorial control of Spo11 alternative splicing by modulation of RNA polymerase II dynamics and splicing factor recruitment during meiosis. Cell death \& disease 11, 1-12 (2020). 
34. Söderholm, S. \& Cantù, C. The WNT/ $\beta$-catenin dependent transcription: A tissue-specific business. WIREs Mechanisms of Disease 13, e1511 (2021).

35. Grossmann, A.H. et al. The small GTPase ARF6 stimulates $\beta$-catenin transcriptional activity during WNT5A-mediated melanoma invasion and metastasis. Science signaling 6, ra14 (2013).

36. Lombardi, A.P. et al. Physiopathological aspects of the Wnt/beta-catenin signaling pathway in the male reproductive system. Spermatogenesis 3, e23181 (2013).

37. Kerr, G.E., Young, J.C., Horvay, K., Abud, H.E. \& Loveland, K.L. Regulated Wnt/beta-catenin signaling sustains adult spermatogenesis in mice. Biology of reproduction 90, 3, 1-12 (2014).

38. Tokue, M. et al. SHISA6 confers resistance to differentiation-promoting Wnt/ $\beta$-catenin signaling in mouse spermatogenic stem cells. Stem Cell Reports 8, 561-575 (2017).

39. Kopera, I.A., Bilinska, B., Cheng, C.Y. \& Mruk, D.D. Sertoli-germ cell junctions in the testis: a review of recent data. Philosophical Transactions of the Royal Society B: Biological Sciences 365, 15931605 (2010).

40. Okada, K. et al. Critical function of RA-GEF-2/Rapgef6, a guanine nucleotide exchange factor for Rap1, in mouse spermatogenesis. Biochemical and biophysical research communications 445, 89-94 (2014).

41. Obholz, K.L., Akopyan, A., Waymire, K.G. \& MacGregor, G.R. FNDC3A is required for adhesion between spermatids and Sertoli cells. Developmental biology 298, 498-513 (2006).

42. Felipe-Medina, N. et al. A missense in HSF2BP causing primary ovarian insufficiency affects meiotic recombination by its novel interactor C190RF57/BRME1. Elife $\mathbf{9}$ (2020).

43. Takemoto, K. et al. Meiosis-Specific C19orf57/4930432K21Rik/BRME1 Modulates Localization of RAD51 and DMC1 to DSBs in Mouse Meiotic Recombination. Cell Reports 31 (2020).

44. Gebauer, F. \& Hentze, M.W. Fertility facts: male and female germ cell development requires translational control by CPEB. Molecular cel/ 8, 247-249 (2001).

45. Yamazaki, T. et al. TCF3 alternative splicing controlled by hnRNP H/F regulates E-cadherin expression and hESC pluripotency. Genes \& development 32, 1161-1174 (2018).

46. Yan, H. et al. Oocyte-derived E-cadherin acts as a multiple functional factor maintaining the primordial follicle pool in mice. Cell death \& disease 10, 160 (2019).

47. Fan, H.-Y. et al. $\beta$-Catenin (CTNNB1) promotes preovulatory follicular development but represses LH-mediated ovulation and luteinization. Molecular endocrinology 24, 1529-1542 (2010). 
48. Uren, P.J. et al. High-throughput analyses of hnRNP H1 dissects its multi-functional aspect. RNA biology 13, 400-411 (2016).

49. White-Cooper, H. \& Davidson, I. Unique aspects of transcription regulation in male germ cells. Cold Spring Harbor perspectives in biology 3, a002626 (2011).

50. Legrand, J.M.D. \& Hobbs, R.M. RNA processing in the male germline: Mechanisms and implications for fertility. Seminars in cell \& developmental biology 79, 80-91 (2018).

51. Licatalosi, D.D. Roles of RNA-binding proteins and post-transcriptional regulation in driving male germ cell development in the mouse, in RNA Processing 123-151 (Springer, 2016).

52. Liu, W. et al. BCAS2 is involved in alternative mRNA splicing in spermatogonia and the transition to meiosis. Nature communications 8, 1-11 (2017).

53. Ji, X. et al. SR proteins collaborate with 7SK and promoter-associated nascent RNA to release paused polymerase. Cell 153, 855-868 (2013).

54. Pintacuda, G. et al. hnRNPK recruits PCGF3/5-PRC1 to the Xist RNA B-repeat to establish polycomb-mediated chromosomal silencing. Molecular cell 68, 955-969. e910 (2017).

55. Kuninger, D.T., Izumi, T., Papaconstantinou, J. \& Mitra, S. Human AP-endonuclease 1 and hnRNP$\mathrm{L}$ interact with a $\mathrm{nCaRE}-$ like repressor element in the AP-endonuclease 1 promoter. Nucleic acids research 30, 823-829 (2002).

56. Wei, C. et al. RBFox2 Binds Nascent RNA to Globally Regulate Polycomb Complex 2 Targeting in Mammalian Genomes. Molecular cell 62, 982 (2016).

57. Xiao, R. et al. Pervasive chromatin-RNA binding protein interactions enable RNA-based regulation of transcription. Cel/ 178, 107-121. e118 (2019).

58. Masuda, T. et al. Cold-inducible RNA-binding protein (Cirp) interacts with Dyrk1b/Mirk and promotes proliferation of immature male germ cells in mice. Proceedings of the National Academy of Sciences 109, 10885-10890 (2012).

59. Zhang, C. et al. The chromatin remodeler Snf2h is essential for oocyte meiotic cell cycle progression. Genes \& development 34, 166-178 (2020).

60. Li, M. et al. G9a-mediated histone methylation regulates cadmium-induced male fertility damage in pubertal mice. Toxicology letters 252, 11-21 (2016).

61. Zhang, Q., Ji, S.-Y., Busayavalasa, K. \& Yu, C. SP016 binds SHOC1 to promote homologous recombination and crossing-over in meiotic prophase I. Science advances (2019). 
62. Peters, A.H. et al. Loss of the Suv39h histone methyltransferases impairs mammalian heterochromatin and genome stability. Cell 107, 323-337 (2001).

63. Takemoto, K. et al. Meiosis-Specific C19orf57/4930432K21Rik/BRME1 modulates localization of RAD51 and DMC1 to DSBs in mouse meiotic recombination. Cell reports 31, 107686 (2020).

64. Felipe-Medina, N. et al. A missense in HSF2BP causing primary ovarian insufficiency affects meiotic recombination by its novel interactor C190RF57/BRME1. Elife 9, e56996 (2020).

65. Masson, S.W., Sorrenson, B., Shepherd, P.R. \& Merry, T.L. $\beta$-catenin regulates muscle glucose transport via actin remodelling and M-cadherin binding. Molecular metabolism 42, 101091 (2020).

66. O'Bryan, M.K. et al. RBM5 is a male germ cell splicing factor and is required for spermatid differentiation and male fertility. PLoS genetics 9 , e1003628 (2013).

67. Dong, J. et al. UHRF1 suppresses retrotransposons and cooperates with PRMT5 and PIWI proteins in male germ cells. Nature communications 10, 1-14 (2019).

68. Wang, X. et al. MFN2 interacts with nuage-associated proteins and is essential for male germ cell development by controlling mRNA fate during spermatogenesis. Development (Cambridge, England) 148, dev196295 (2021).

\section{Figures}


a
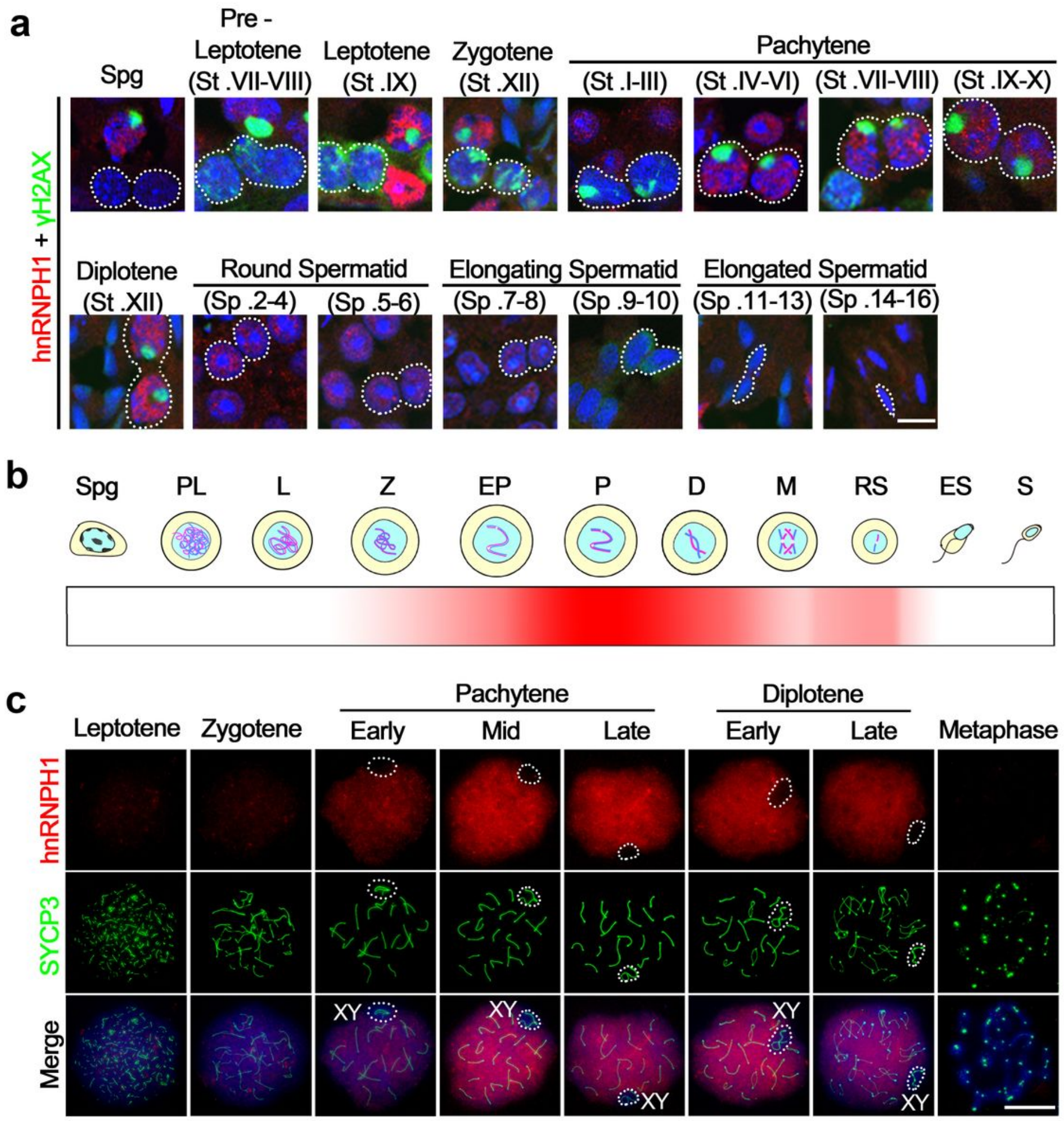

Figure 1

hnRNPH1 displays a dynamic expression pattern during spermatogenesis. (a) Anti-hnRNPH1 and anti$\mathrm{YH} 2 \mathrm{AX}$ antibodies were used to double immunostaining of wild-type (WT) germ cells from adult testicular cryosections. The dotted lines outline the indicated cell types. Scale bars $=10 \mu \mathrm{m}$. (b) Time dynamic expression pattern of hnRNPH1 in adult testis during spermatogenesis. Spg, spermatogonia; PL, preleptotene; L, leptotene; Z, zygotene; EP, early pachytene; P, pachytene; D, diplotene; RS, round spermatids; 
ES, elongating spermatids; S, spermatozoa. (c) Double immunostaining with hnRNPH1 and SYCP3 on surface-spread spermatocytes from WT adult mice are shown. The dotted lines outline XY-body. Scale bars $=5 \mu \mathrm{m}$.

a

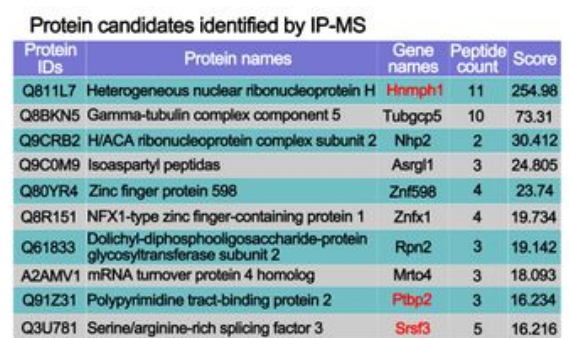

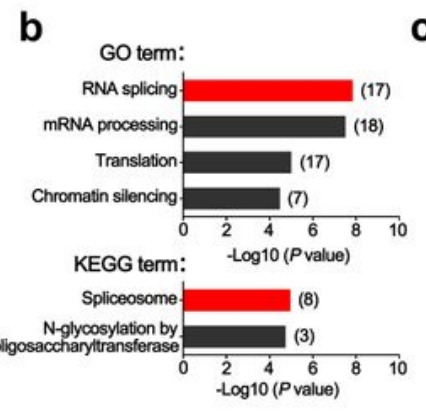

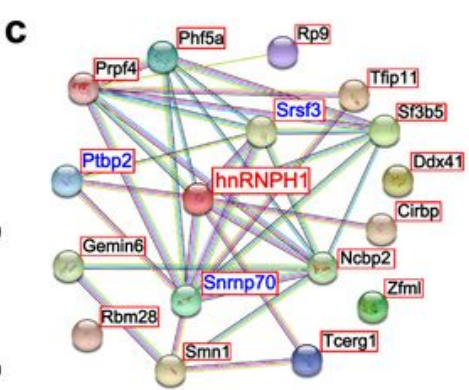

d

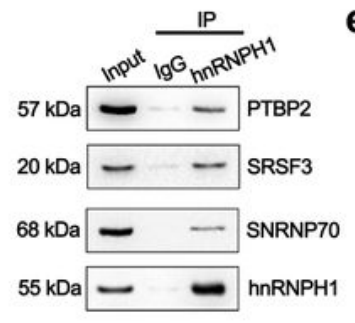

e

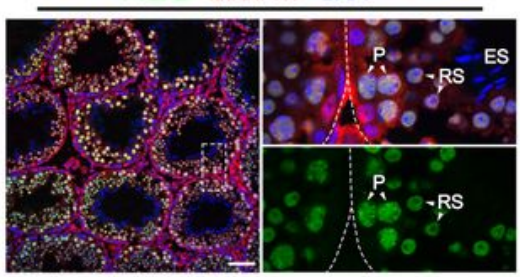

SRSF3 + hnRNPH1 + DAPI

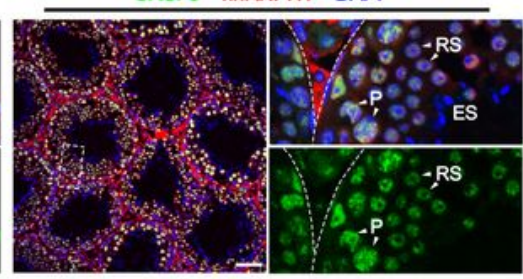

$\mathbf{f}$
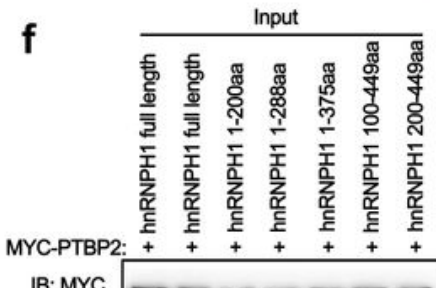

IB: MYC
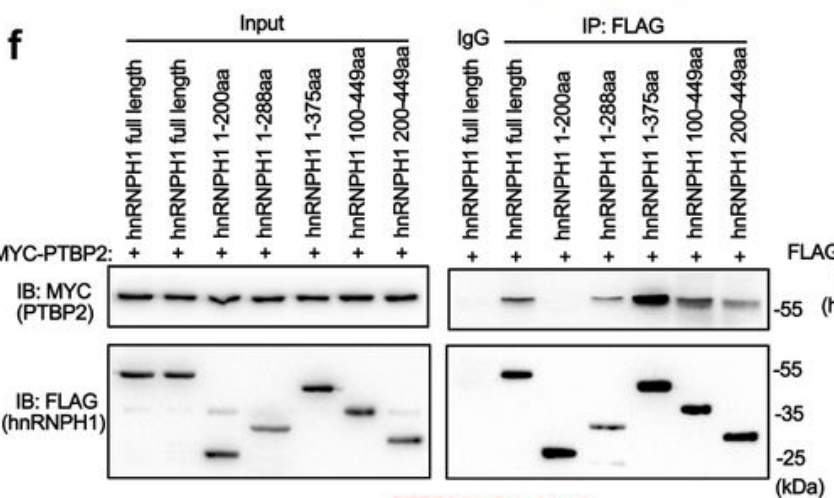

PTBP2 binding domain

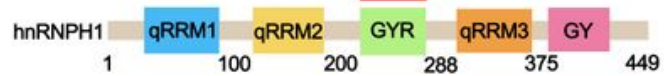

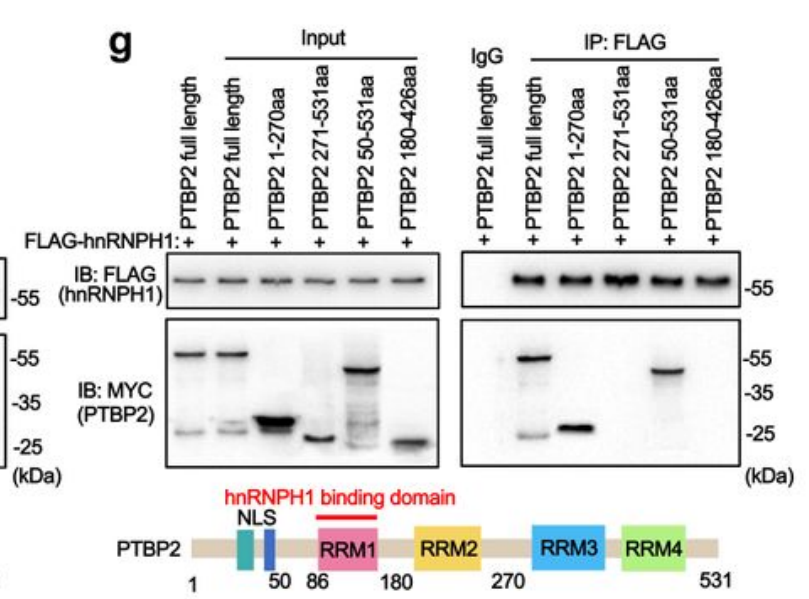

h
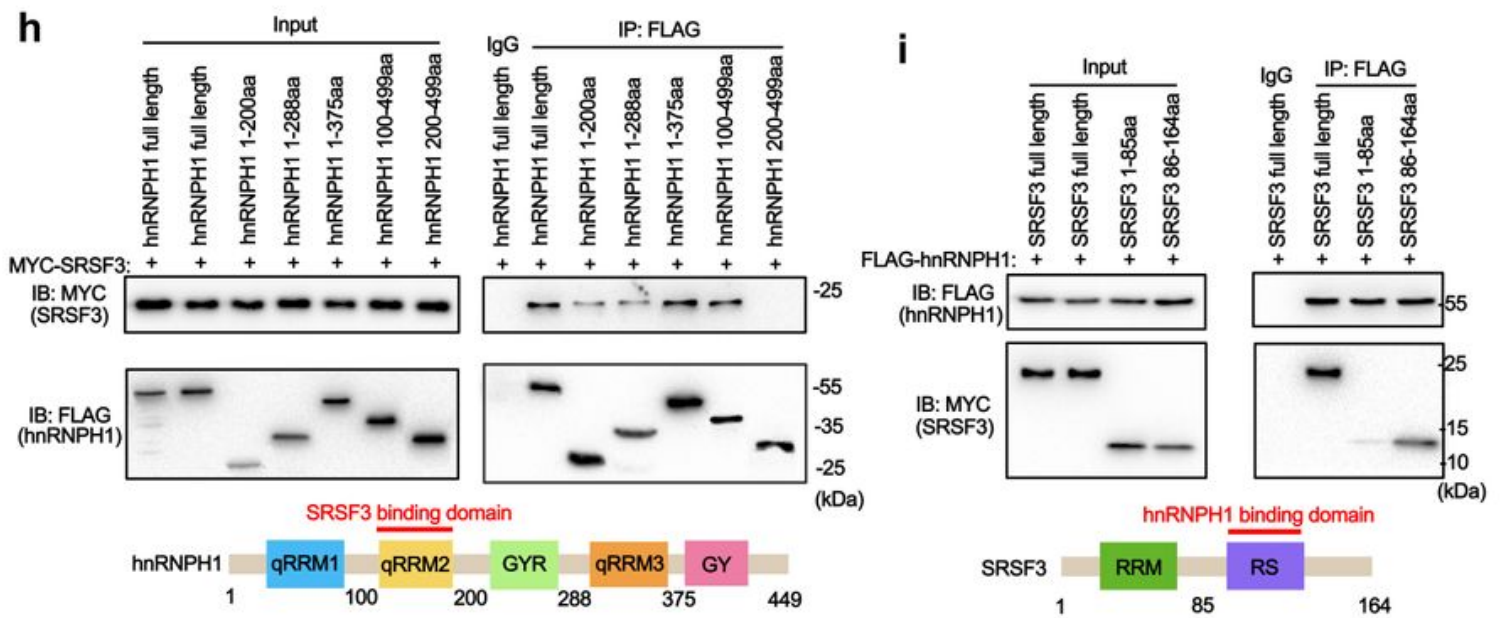

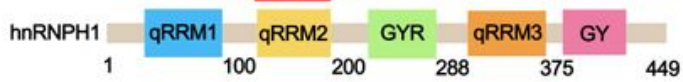

Figure 2

hnRNPH1 interacts with RNA splicing factors PTBP2 and SRSF3. (a) A list of 10 hnRNPH1-interacting partners in mouse adult WT testes identified by IP-MS is shown. (b) GO and KEGG term enrichment 
analyses showing the hnRNPH1-interacting proteins identified from IP-MS data. (c) The String 10.5 program (http://string-db.org) analyses showing the protein interaction networks among 17 candidate proteins associated with mRNA splicing. (d) Validation of interactions between hnRNPH1 and three putative hnRNPH1-interacting proteins (PTBP2, SRSF3, and SNRNP70) in mouse testes by in vivo coimmunoprecipitation (Co-IP) assays are shown. IgG was used as a negative control. (e) Coimmunofluorescence staining of PTBP2 (left panels) and SRSF3 (right panels) with hnRNPH1 in adult mouse testes. P, pachytene spermatocytes; RS, round spermatids; ES, elongating spermatids. DNA was stained with DAPI. Scale bars $=50 \mu \mathrm{m}$. (f-i) Reciprocal Co-IP assays of interaction domains between hnRNPH1 and its binding partners PTBP2 and SRSF3. HEK293T cells were co-transfected with MYCPTBP2 and the indicated fragments of FALG-hnRNPH1(f), or co-transfected with FALG-hnRNPH1 and the indicated fragments of MYC-PTBP2 (g), or co-transfected with MYC-SRSF3 and the indicated fragments of FALG-hnRNPH1(h), or co-transfected with FALG-hnRNPH1 and the indicated fragments of MYC-SRSF3 (i), immunoprecipitated with anti-FLAG antibody, and immunoblotted with FLAG and MYC antibodies, respectively.

a
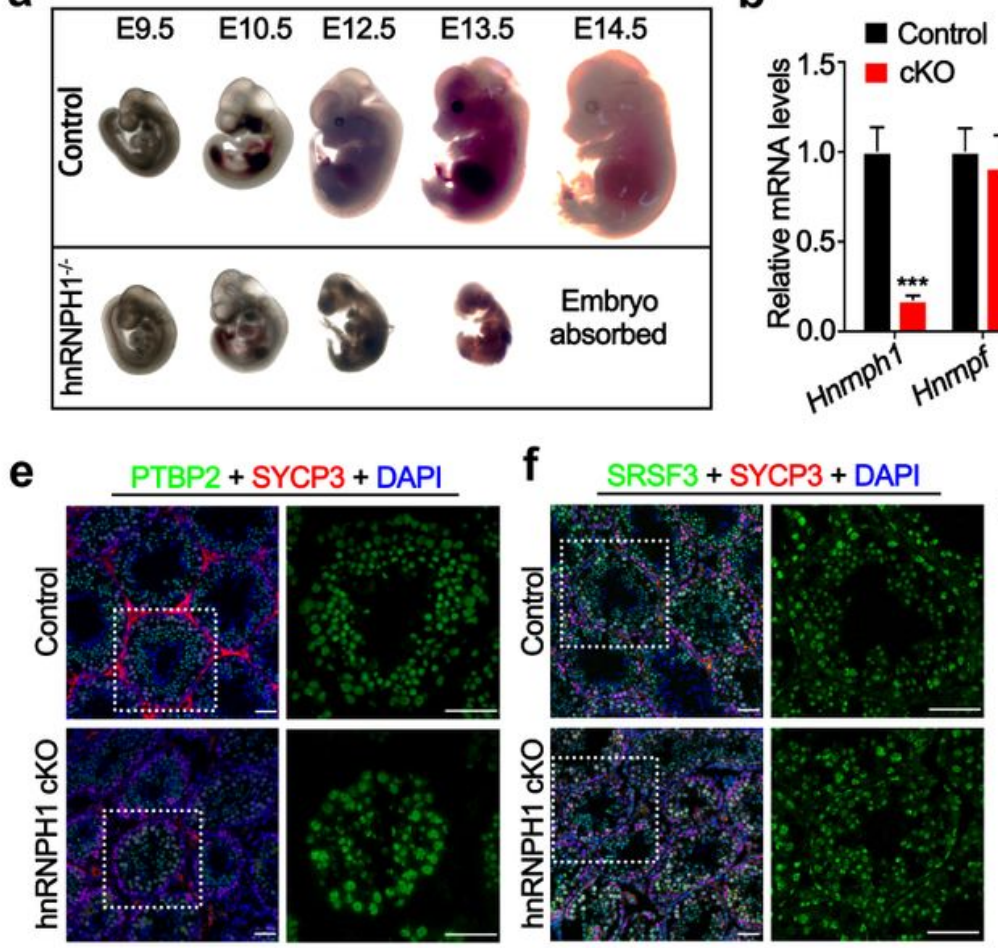

i
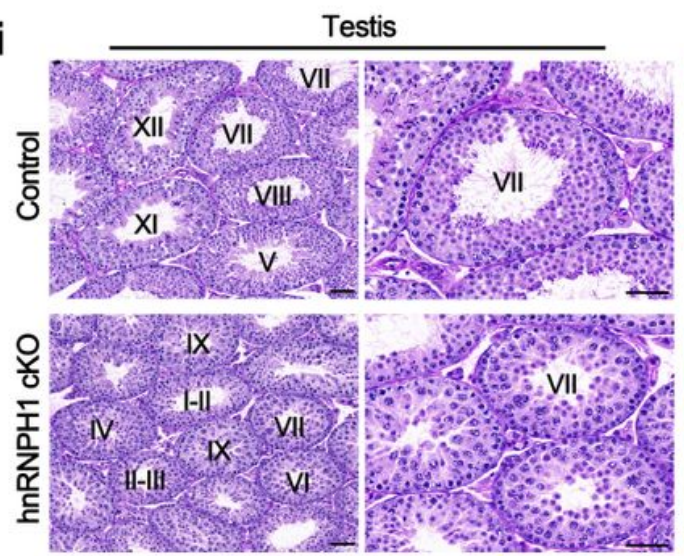

b

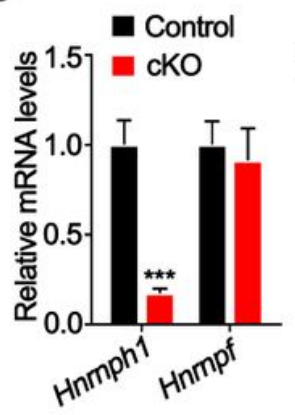

9

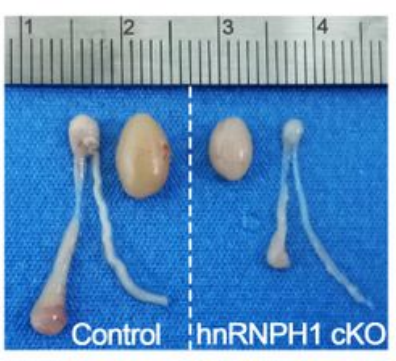

d

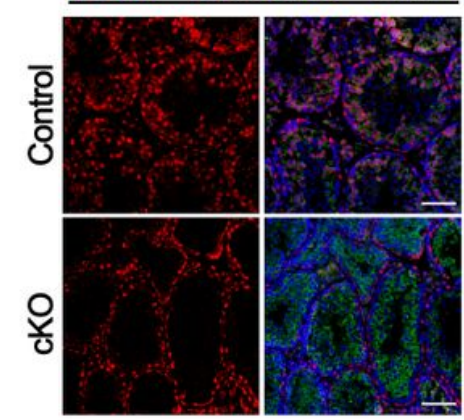

h

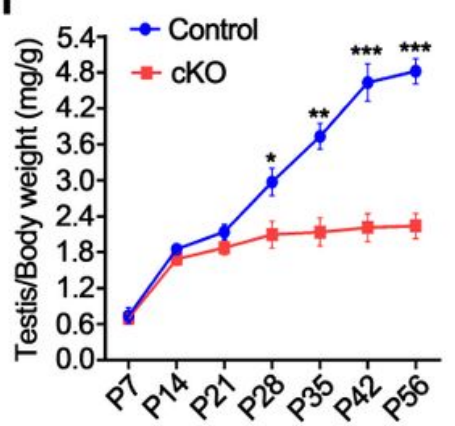

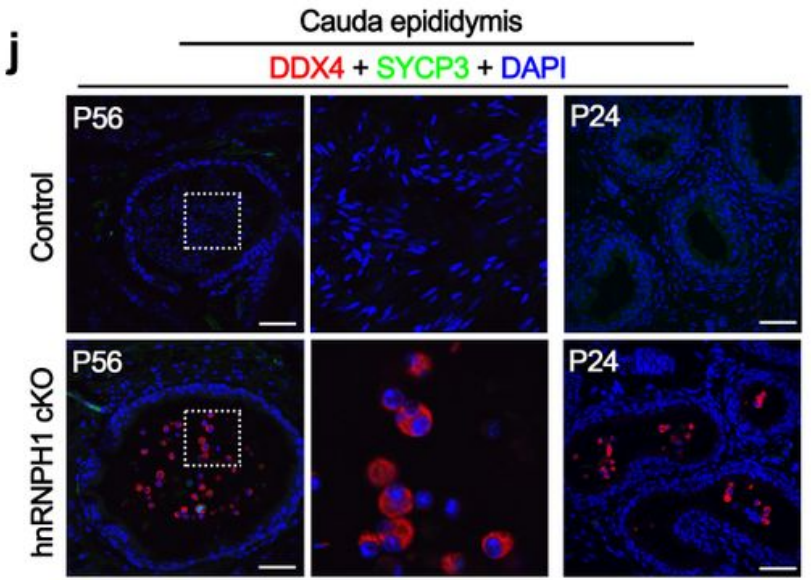




\section{Figure 3}

hnRNPH1 is indispensable for embryonic development and male fertility in mice. (a) Representative embryos at various developmental time points, including embryonic day 9.5 (E9.5), E10.5, E 12.5, E 13.5, and $\mathrm{E}$ 14.5, as derived from control and hnRNPH1 -/- mice. (b) Real-time qPCR analyses of Hnrnph1 and Hnrnpf mRNA levels in control and hnRNPH1 cKO testes of adult mice. Data are presented as mean \pm SD, $\mathrm{n}=3 .{ }^{\star * *} \mathrm{P}<0.001$ by Student's t-test. (c) Western blotting analyses of hnRNPH1 and hnRNPF protein in control and hnRNPH1 cKO testes of adult mice. GAPDH was used as a loading control. (d) Immunofluorescence (IF) staining of hnRNPH1 in control and hnRNPH1 cKO testes of adult mice. DDX4 was co-stained to indicate the location of the germ cell. The DNA was stained with DAPI. Scale bars $=50$ $\mu \mathrm{m}$. (e-f) Co-immunofluorescence staining of PTBP2 (e) and SRSF3 (f) with SYCP3 in testis sections from adult control and hnRNPH1 cKO mice. The DNA was stained with DAPI. Scale bars $=50 \mu \mathrm{m}$. (g) Gross morphology of the testes and the epididymides from adult control and hnRNPH1 cKO mice. (h) Testis growth curves of control and hnRNPH1 cKO mice from postnatal day 7 (P7) to P56. Data are presented as mean $\pm S D, n=3 .{ }^{*}<<0.05, * * P<0.01,{ }^{\star} * * P<0.001$ by Student's t-test. (i) Periodic acid-Schiff (PAS) staining of testes and epididymides from control and hnRNPH1 cKO mice at P56. Scale bars $=50 \mu \mathrm{m}$. (j) Co-immunofluorescent staining of DDX4 with SYCP3 on control and hnRNPH1 cKO cauda epididymidis sections at P56 and P24, respectively. Scale bars $=50 \mu \mathrm{m}$. 
a

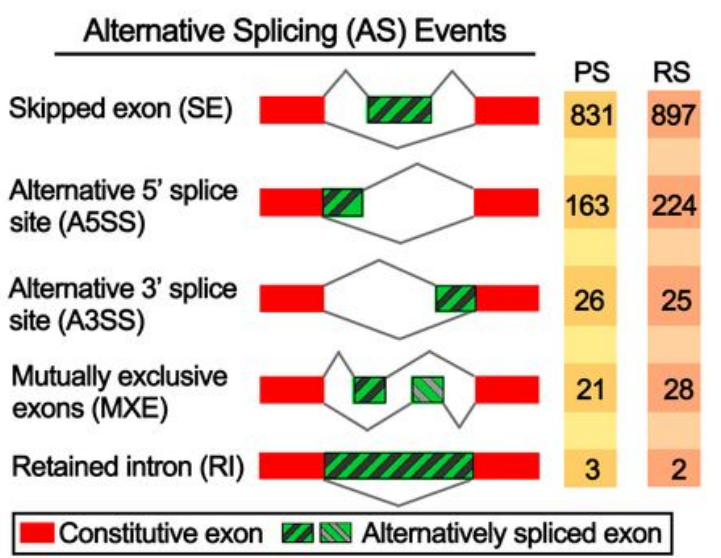

b

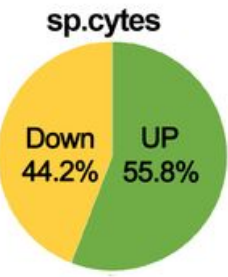

sp.cytes

C

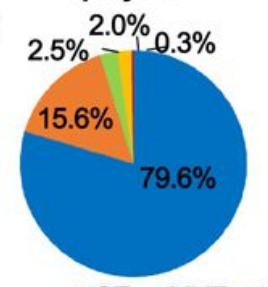

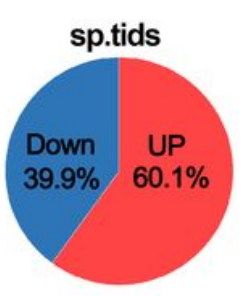

sp.tids

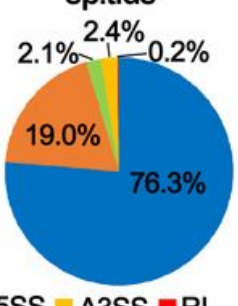

d

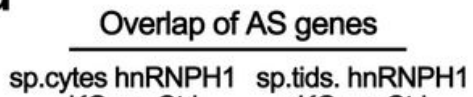

sp.cytes hnRNPH1 sp.tids. hnRNPH1 cKO vs. Ctrl

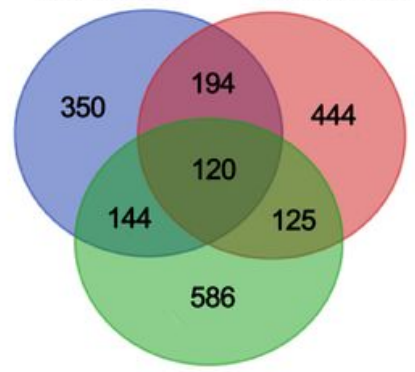

Ptbp2 cKO vs. Ctrl

e
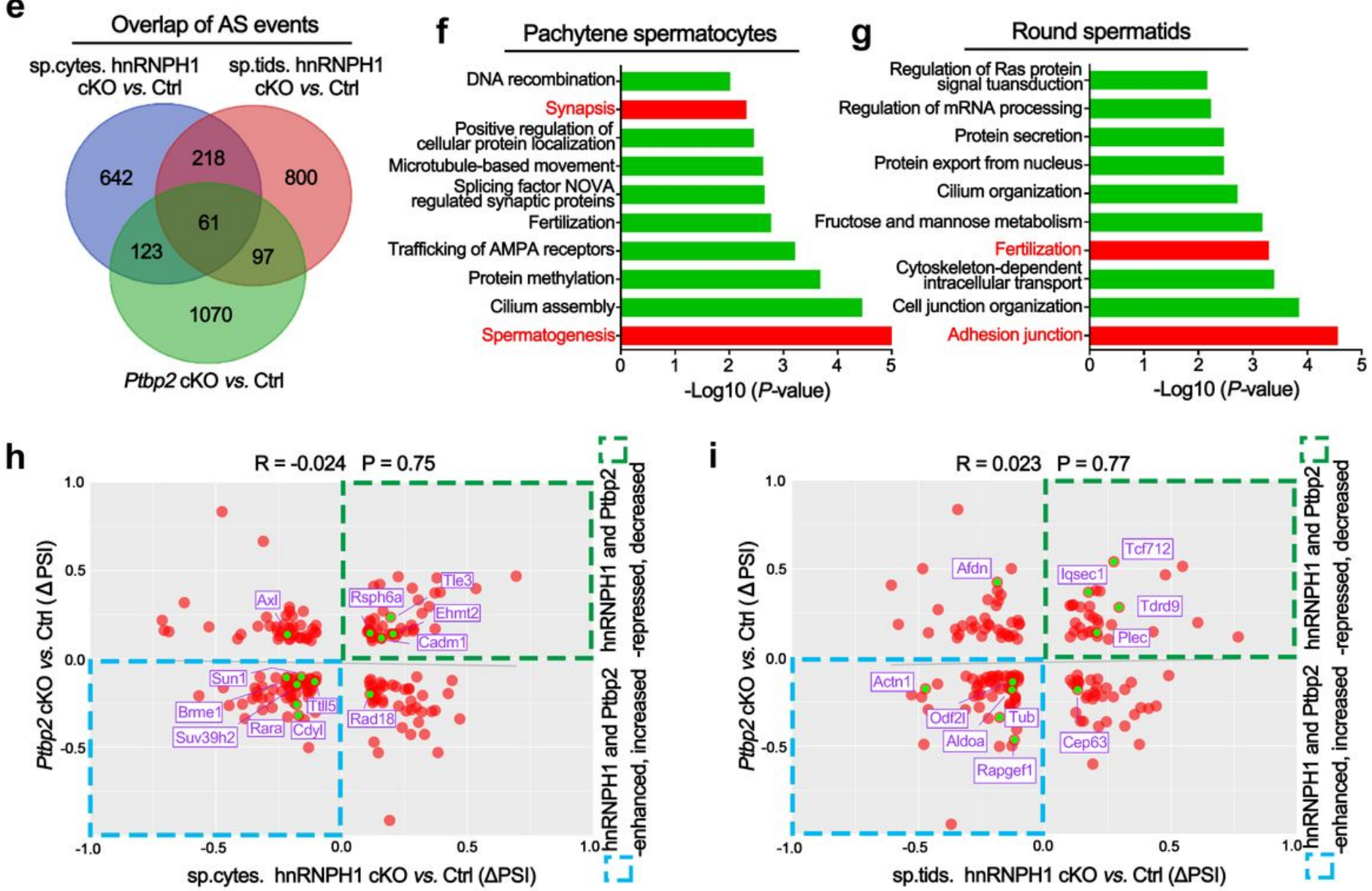

\section{Figure 4}

hnRNPH1 and PTBP2 synergistically regulate pre-mRNA splicing in spermatogenic cells. (a) Summary of differential splicing analysis performed using pachytene spermatocytes (PS) and round spermatids (RS) isolated from the control and hnRNPH1 cKO testis at P25. Numbers of predicted alternative splicing (AS) events in each category upon hnRNPH1 deletion are indicated. (b) Pie charts showing percentages of changed AS events identified in hnRNPH1 cKO versus control spermatocytes (left) and spermatids (right). (c) Pie charts representing the distribution of regulated splicing events among different splicing patterns 
in hnRNPH1 cKO versus control spermatocytes (left) and spermatids (right). (d) Venn diagrams showing overlap of abnormal AS genes (808) in hnRNPH1 cKO spermatocytes, abnormal AS genes (783) in hnRNPH1 cKO spermatids, and abnormal AS genes (975) in Ptbp2 cKO testis. (e) Venn diagrams showing overlap of abnormal AS events (1044) in hnRNPH1 cKO spermatocytes, abnormal AS events (1176) in hnRNPH1 cKO spermatids, and abnormal AS events (1351) in Ptbp2 cKO testis. (f-g) GO term enrichment analyses of genes with the same abnormal AS events caused by hnRNPH1- and PTBP2ablation in spermatocytes $(\mathrm{f})$ and spermatids $(\mathrm{g})$. The top 10 biological processes ranked by log10 ( $\mathrm{P}-$ value) are listed. (h-i) Distribution of $\triangle \mathrm{PSI}$ values for genes with abnormal AS events in hnRNPH1 cKO spermatocytes $(\mathrm{h})$ and spermatids (i). The genes involved in indicated signaling pathways and coregulated by hnRNPH1 and PTBP2 were marked in red. 


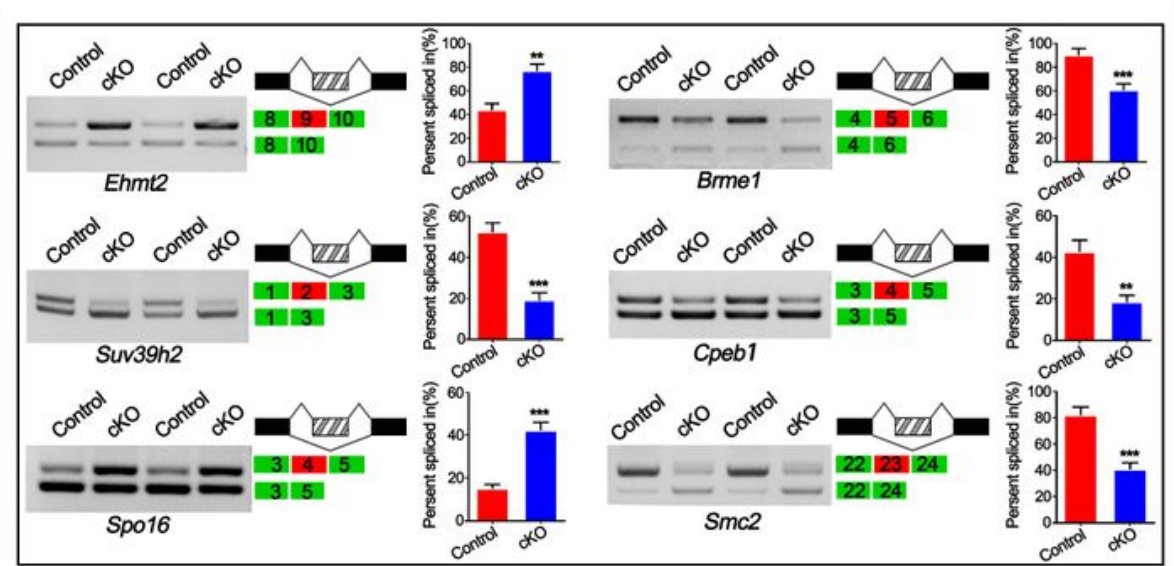

b
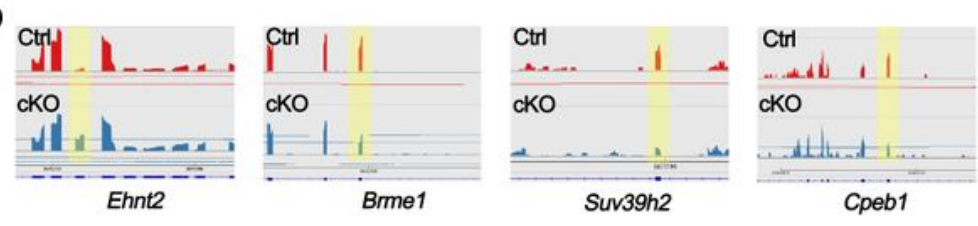

Cpeb1

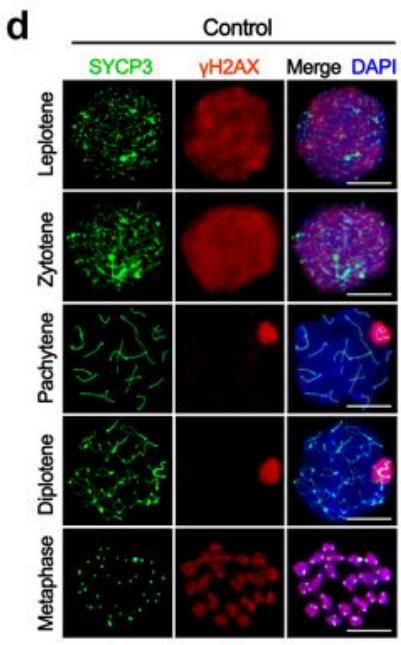

e

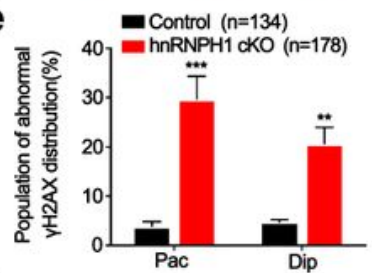

f

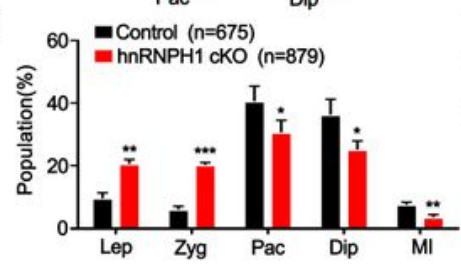

hnRNPH1 cKO

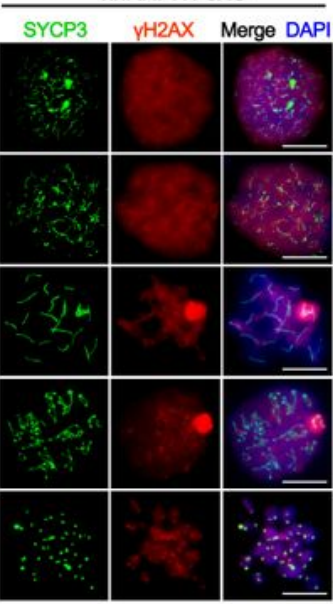

h

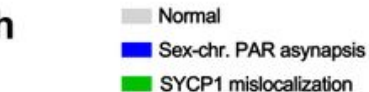

- SYCP1 mislocalization

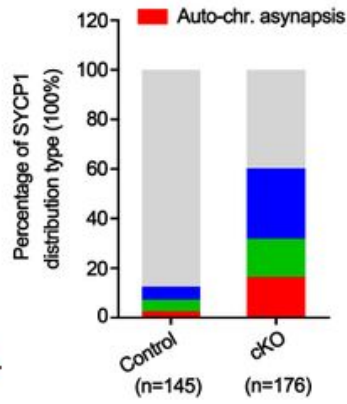

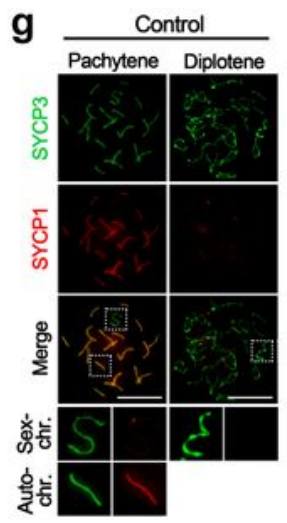

j

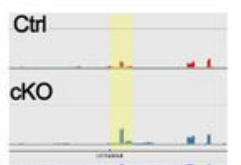

Spo16
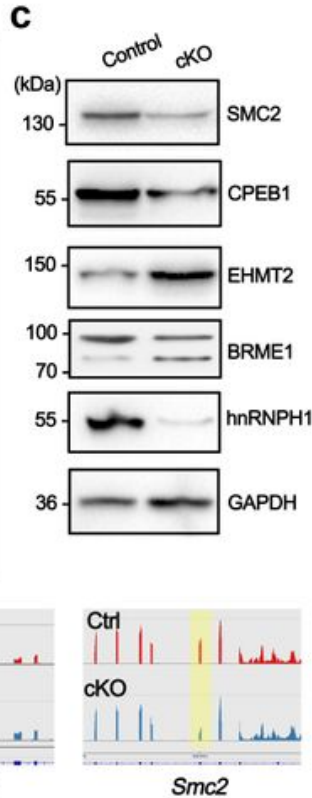

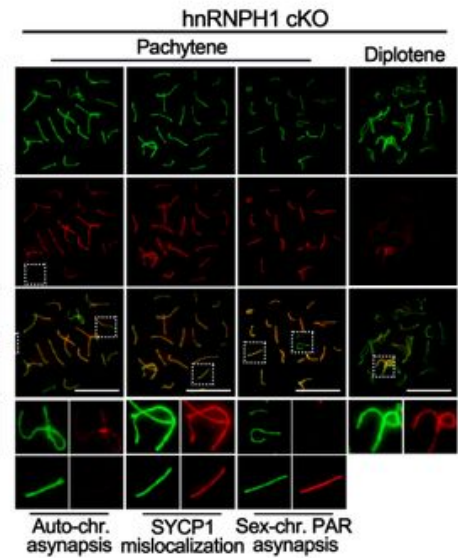

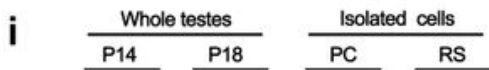

Ctr cKO Ctrl cKO Ctrl cKO Ctrl CKO
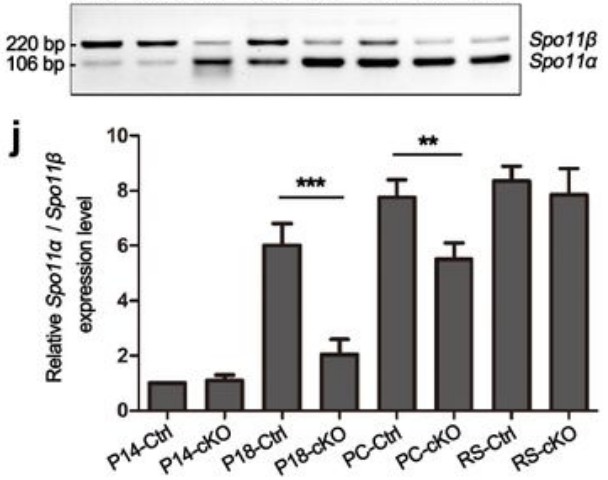

Figure 5

hnRNPH1 deficiency in male germ cells causes aberrant AS of meiotic-related genes and defective meiosis. (a) Representative examples of RT-PCR analyses for indicated AS events differentially regulated genes between control and hnRNPH1 cKO pachytene spermatocytes isolated from P25 mice are shown. Middle panels represent the schematic diagram of AS exons detected by RNA-seq analysis. Right panels show the quantification of percent spliced in (PSI); $n=3$. (b) RNA-seq results of alternative sites in genes 
related to meiosis using IGV software analysis. Splicing sites are indicated by the yellow box. (c) Western blotting analyses of the expression of SMC2, CPEB1, EHMT2, BRME1, and hnRNPH1 in control and hnRNPH1 cKO spermatocytes isolated from P25 mice. GAPDH was used as a loading control. (d) Coimmunofluorescence staining of SYCP3 with $\mathrm{YH} 2 \mathrm{AX}$ in spermatocyte spreads from control and hnRNPH1 cKO mice. Scale bar $=5 \mu \mathrm{m}$. (e) Percentages of abnormal $\mathrm{YH} 2 \mathrm{AX}$ distribution at the pachytene (Pac) and diplotene (Dip) stages in the control and hnRNPH1 cKO male mice. Data are presented as mean $\pm \mathrm{SD}$. **P $\leq 0.01,{ }^{\star * *} P \leq 0.001$ by Student's t-test. Biologically independent mice $(n=3)$ for each genotype were examined. (f) Percentages of spermatocytes at the leptotene (Lep), zygotene (Zyg), pachytene (Pac), diplotene (Dip) and metaphase (MI) stages in the control and hnRNPH1 cKO mice. Data are presented as mean $\pm S D$. ${ }^{*} P \leq 0.05,{ }^{*} P \leq 0.01$ by Student's t-test. Biologically independent mice $(n=3)$ for each genotype were examined. (g) Co-immunofluorescence staining of SYCP3 with SYCP1 in spermatocyte spreads at pachytene and diplotene stages from control and hnRNPH1 cKO male mice. Scale bars = 5 $\mu \mathrm{m}$. (h) Percentages of SYCP1 distribution types in pachytene spermatocytes from the control and hnRNPH1 cKO mice. Biologically independent mice $(n=3)$ for each genotype were examined. (i) RT-PCR analyses of Spo11 $\beta$ and Spo11a expression levels in control and hnRNPH1 cKO whole testes at the P14 and P18, as well as spermatocytes and round spermatids isolated from control and hnRNPH1 cKO mice. (j) Relative Spo11a / Spo11 $\beta$ expression level in indicated groups are shown. Data are presented as mean $\pm S D, n=3 .{ }^{\star *} P \leq 0.01,{ }^{\star \star *} P \leq 0.001$ by Student's t-test. 
a

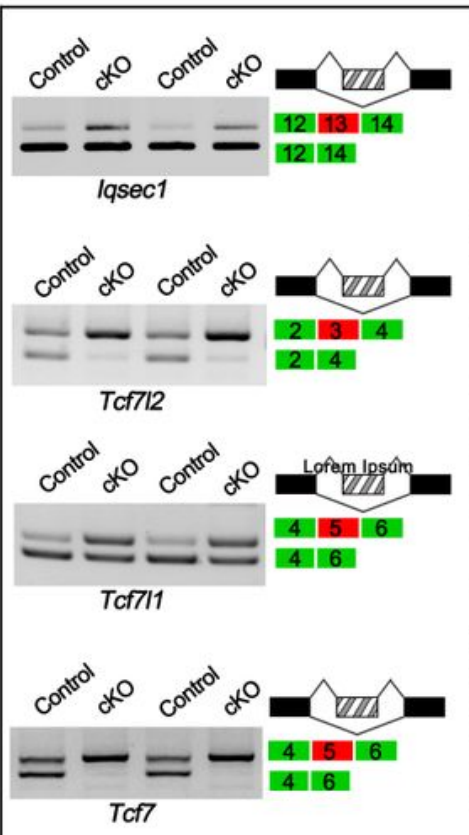

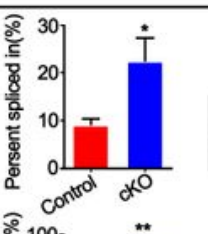
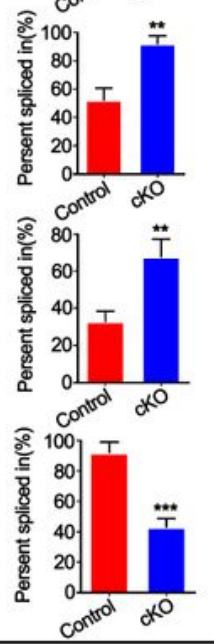

C
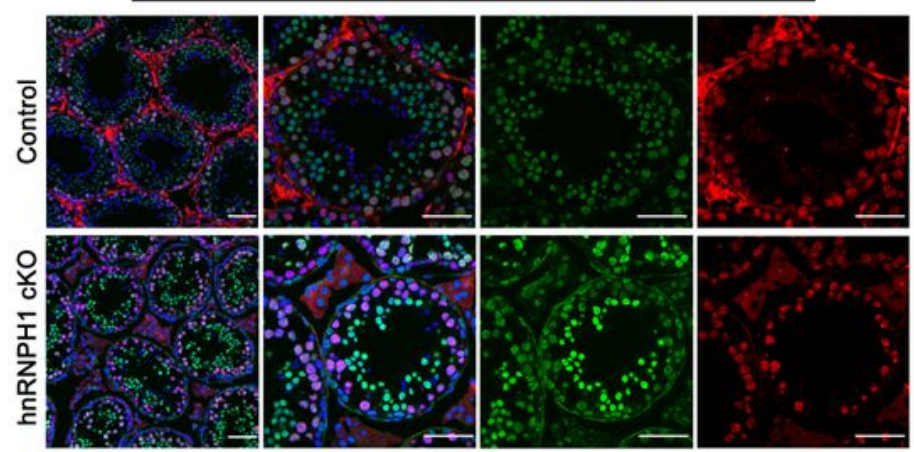

e

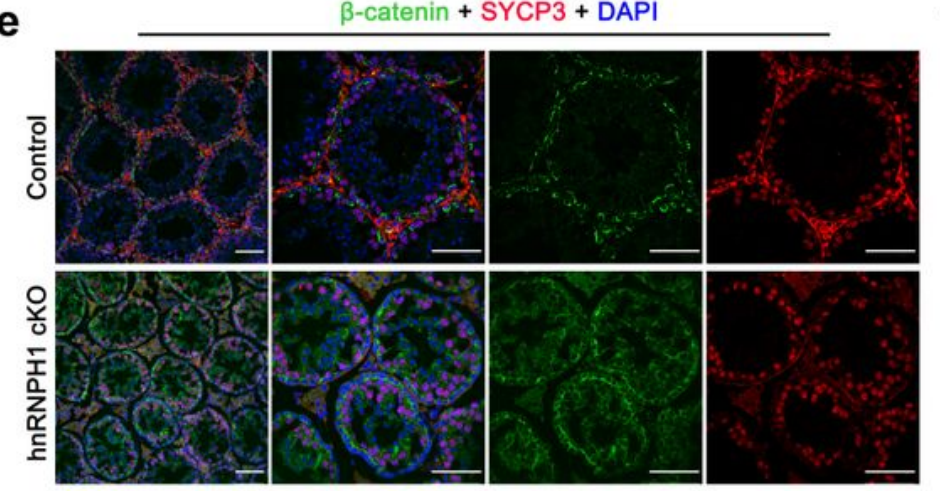

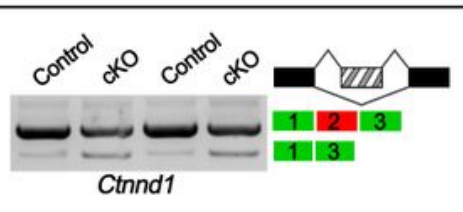
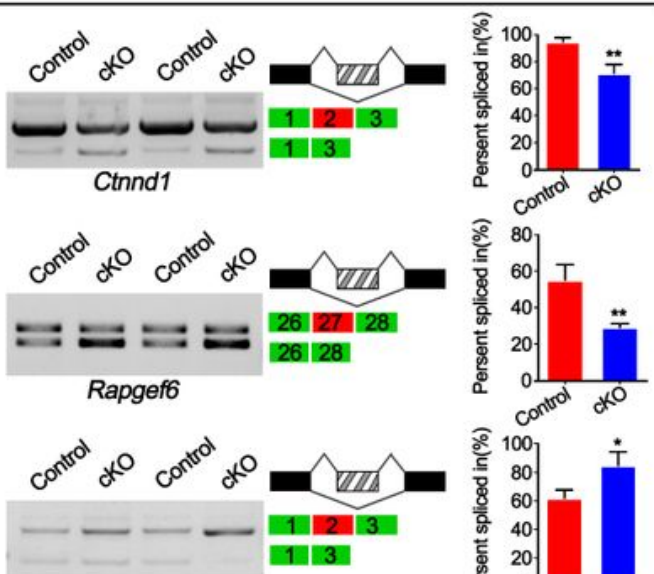

Fndc3a

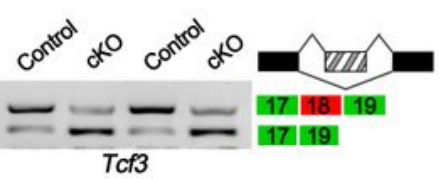

b

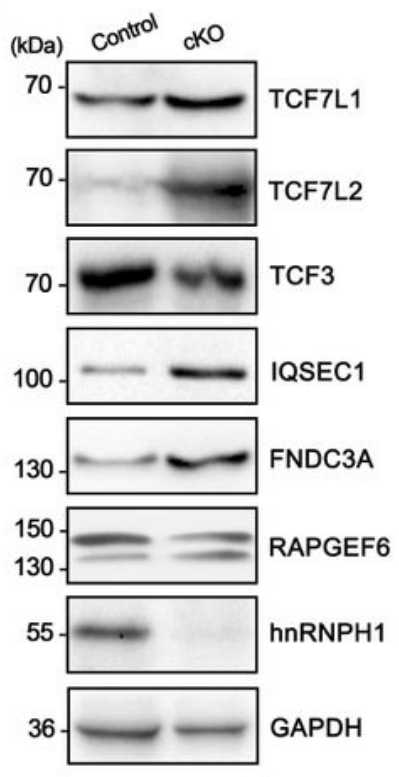

d $\quad$ TCF7L2 + SYCP3 + DAPI

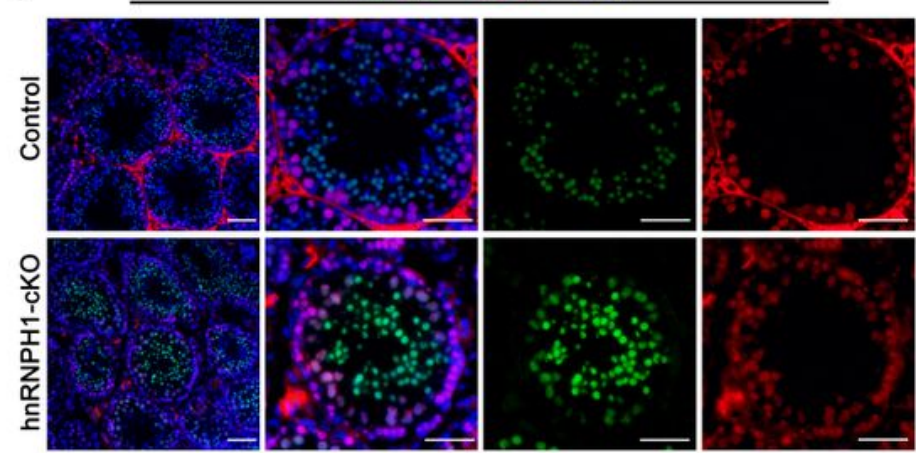

f
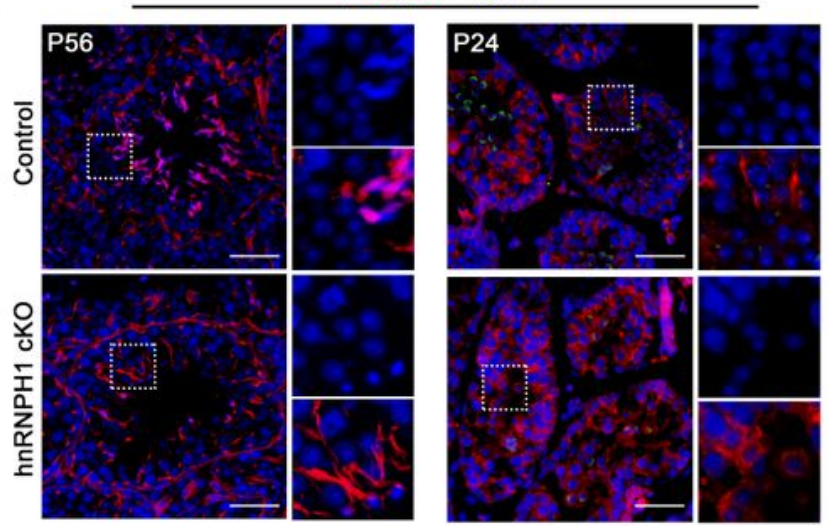

\section{Figure 6}

Ablation of hnRNPH1 in germ cells results in abnormal AS of cell junction-related genes and defective germ-Sertoli cell communications. (a) Representative examples of RT-PCR analyses for indicated AS events differentially regulated genes between control and hnRNPH1 cKO round spermatids are shown. Middle panels represent the schematic diagram of alternatively spliced exons detected by RNA-Seq analysis. Right panels show the quantification of percent spliced in (PSI). Data are presented as mean \pm 
$S D, n=3 .{ }^{*} P \leq 0.05,{ }^{* *} P \leq 0.01,{ }^{* \star * P} \leq 0.001$ by Student's t-test. (b) Western blotting analyses of the expression of TCF7L1, TCF7L2, TCF3, IQSEC1, FNDC3A, RAPGEF6, and hnRNPH1 in control and hnRNPH1 cKO round spermatids isolated from P25 mice. GAPDH was used as a loading control. (c-e) Coimmunofluorescence staining of SYCP3 with TCF7L1 (c), TCF7L2 (d), and $\beta$-catenin (e) on testis section from control and hnRNPH1 cKO mice at P56. Scale bars $=50 \mu \mathrm{m}$. (f) Fluorescence microscopy to detect F-ACTIN (phalloidin) and DNA (DAPI) in testes from control and hnRNPH1 cKO mice at P56 (left panels) and P24 (right panels). Scale bars $=50 \mu \mathrm{m}$.

a

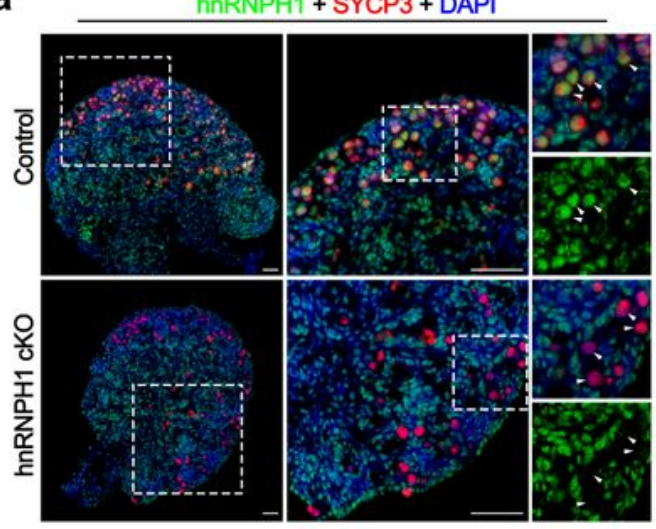

c

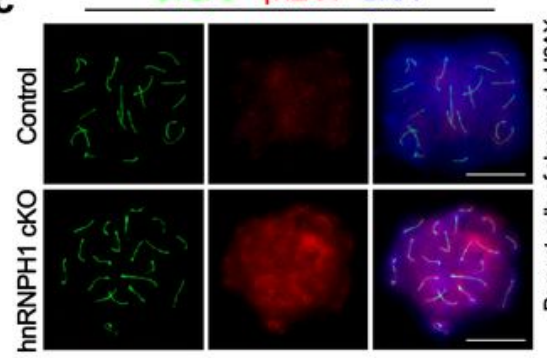

e

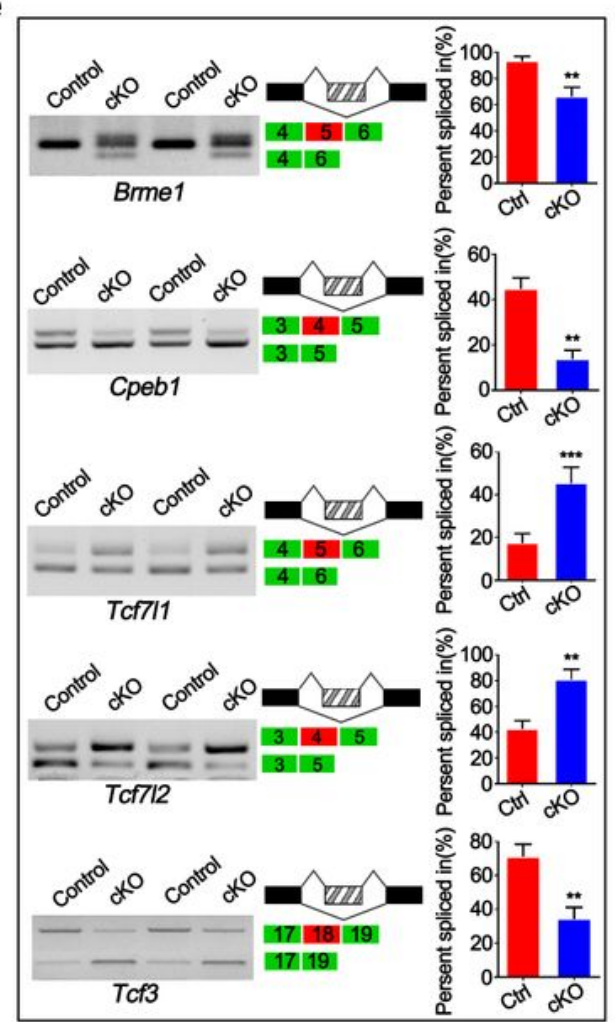

b
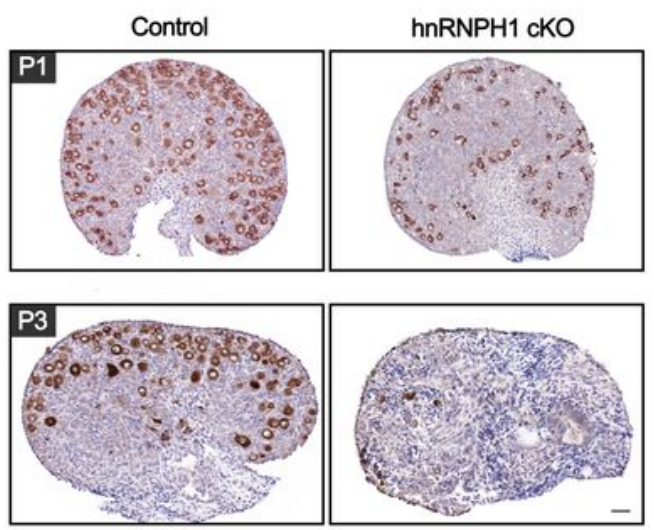

d
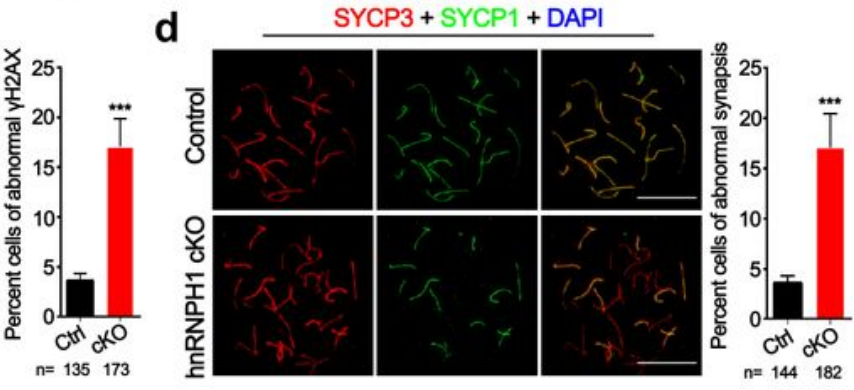

f $\underline{\mathrm{TCF} 7 \mathrm{~L} 1+\mathrm{SYCP} 3+\mathrm{DAPI}}$

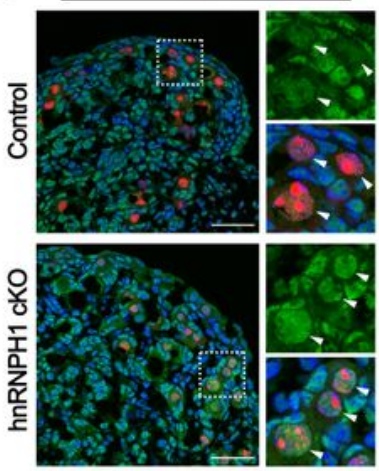

h $\beta$-catenin + SYCP3 + DAPI

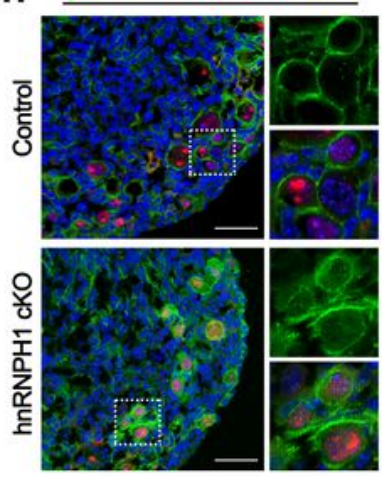

g $\underline{\mathrm{TCF} 7 L 2+\mathrm{SYCP} 3+\mathrm{DAPI}}$

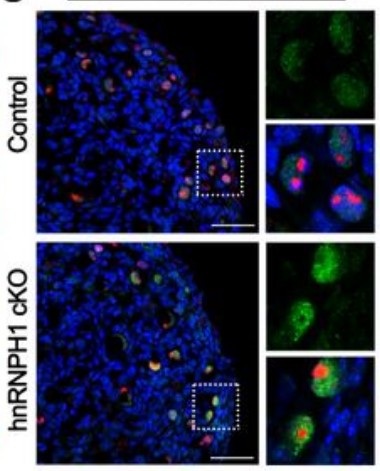

E-cadherin + SYCP3 + DAPI
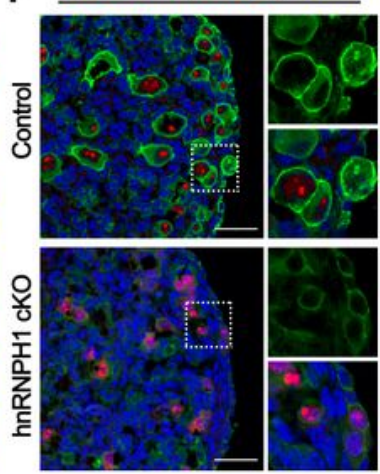


\section{Figure 7}

hnRNPH 1 is essential for the meiosis processes of early oocytes and female fertility. (a) Coimmunofluorescence staining of hnRNPH1 and SYCP3 in ovaries from control and hnRNPH1 cKO mice at E17.5. Scale bars $=50 \mu \mathrm{m}$. Oocytes are indicated with white arrow heads. (b) Immunohistochemical staining of DDX4 in control and hnRNPH1 cKO ovaries at P1 and P3. Scale bars $=50 \mu \mathrm{m}$. (c) Coimmunofluorescence staining of SYCP3 with $\mathrm{YH} 2 \mathrm{AX}$ in chromosome spread of pachytene oocytes from control and hnRNPH1 $\mathrm{cKO}$ mice at E17.5 is shown. The right histogram shows the quantification of the percentage of the cells with abnormal $\mathrm{yH} 2 \mathrm{AX}$ signals. Scale bars $=5 \mu \mathrm{m}$. Data are presented as mean \pm SD. ${ }^{* \star *} P \leq 0.001$ by Student's t-test. Biologically independent mice $(n=3)$ for each genotype were examined. (d) Co-immunofluorescence staining of SYCP3 with SYCP1 in chromosome spread of pachytene oocytes from control and hnRNPH1 cKO mice at E17.5 is shown. The right histogram shows the quantification of the percentage of the cells with abnormal synapsis. Scale bars $=5 \mu \mathrm{m}$. Data are presented as mean \pm SD. ${ }^{* \star *} P \leq 0.001$ by Student's t-test. Biologically independent mice $(n=3)$ for each genotype were examined. (e) Representative examples of RT-PCR analyses for indicated AS events differentially regulated genes between control and hnRNPH1 cKO ovaries at E17.5 are shown. Middle panels represent the schematic diagram of alternatively spliced exons. Right panels show the quantification of percent spliced in (PSI). Data are presented as mean $\pm S D, n=3$. ${ }^{\star \star} P \leq 0.01$, ${ }^{\star \star *} P \leq$ 0.001 by Student's t-test. (f-i) Co-immunofluorescence staining of SYCP3 with TCF7L1 (f), TCF7L2 (g), $\beta$ catenin (h), and E-cadherin (i) in ovaries from control and hnRNPH1 cKO mice at P0. Scale bars $=50 \mu \mathrm{m}$. 
a

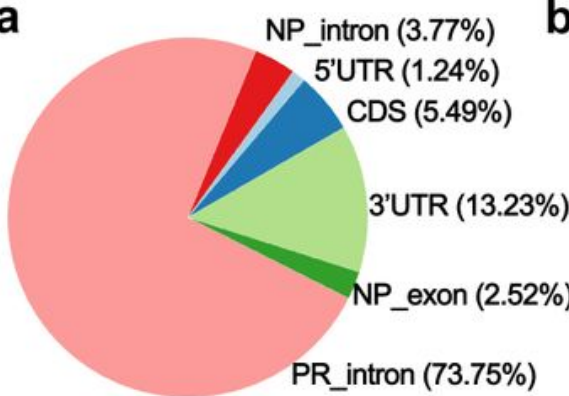

b

\begin{tabular}{|c|c|c|c|c|}
\hline Rank & Motif & P-value & $\log P$-value & 6 of Target \\
\hline 1 & GCACCG & $1.00 E-15$ & $-3.53 E+1$ & $58.11 \%$ \\
\hline 2 & 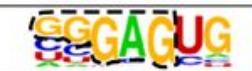 & $1.00 \mathrm{E}-12$ & $-3.53 E+1$ & $66.64 \%$ \\
\hline 3 & GCGCCG & $1.00 E-11$ & $-2.54 \mathrm{E}+1$ & $44.15 \%$ \\
\hline 4 & GCUGGAC & $1.00 E-10$ & $-2.53 E+1$ & $80.37 \%$ \\
\hline 5 & ЧGCUGGCC & $1.00 E-10$ & $-2.51 E+1$ & $65.33 \%$ \\
\hline
\end{tabular}

C

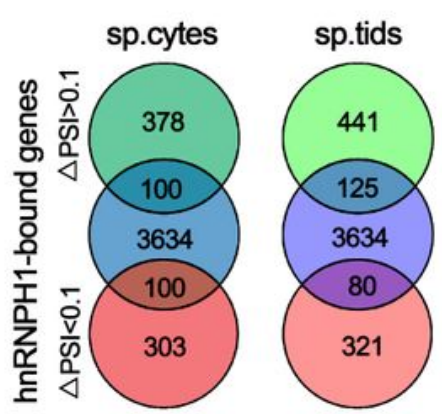

d

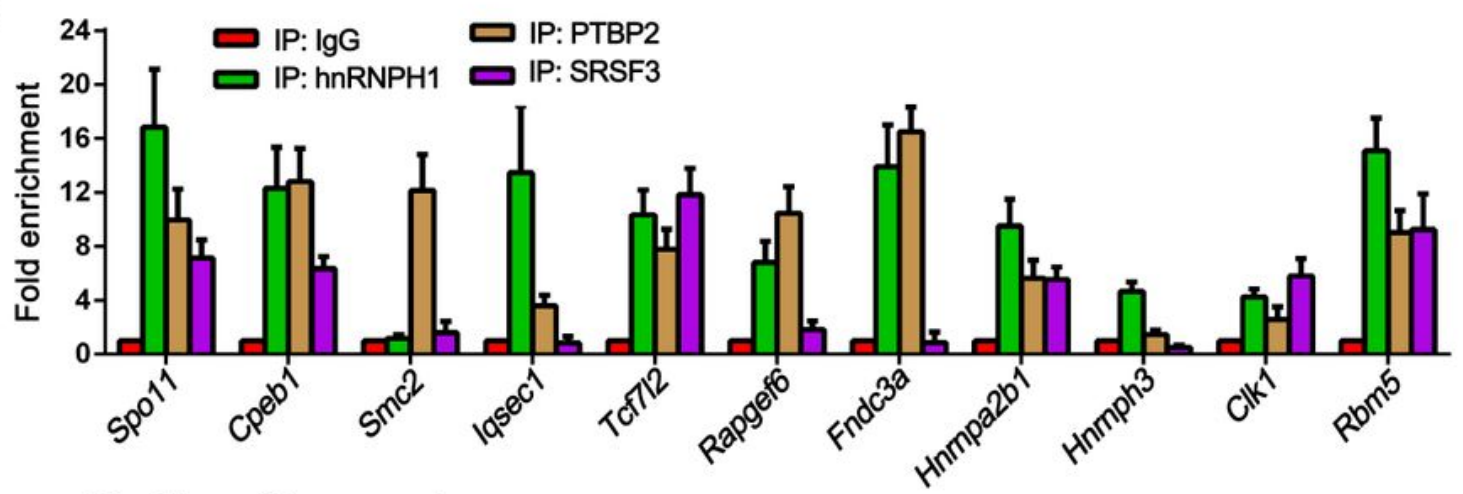

e

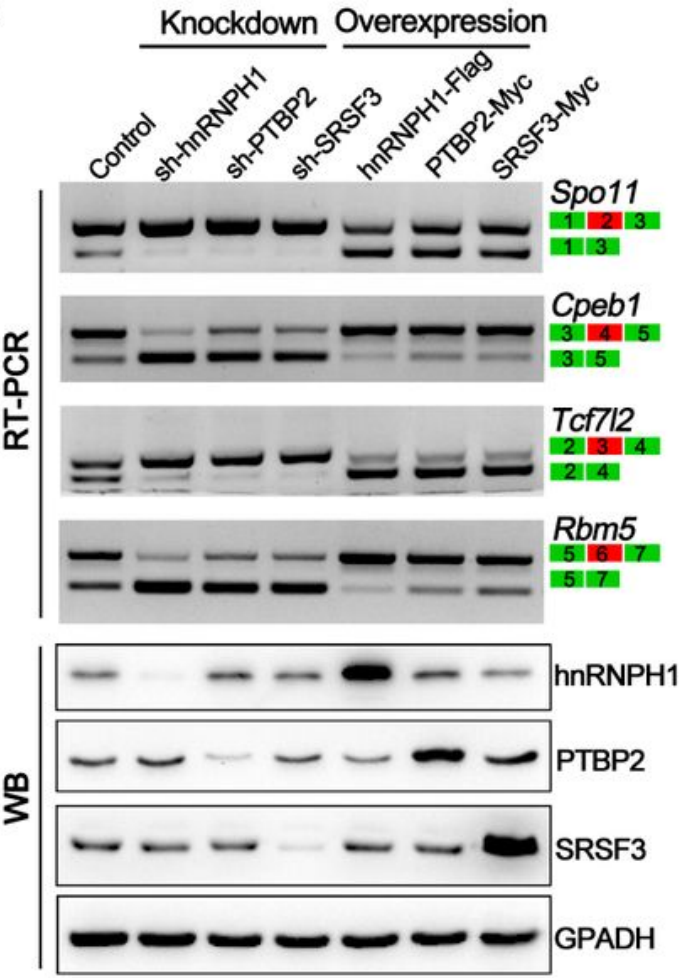

f

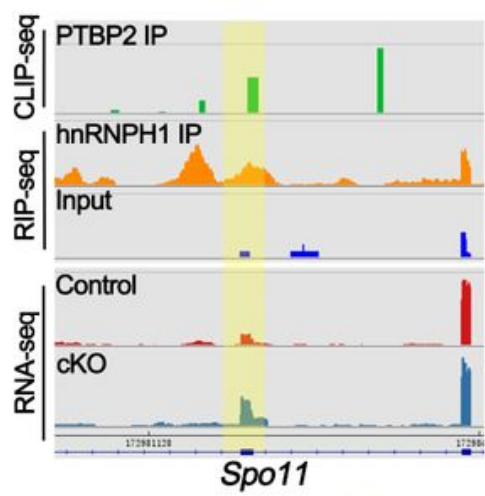

|

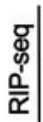

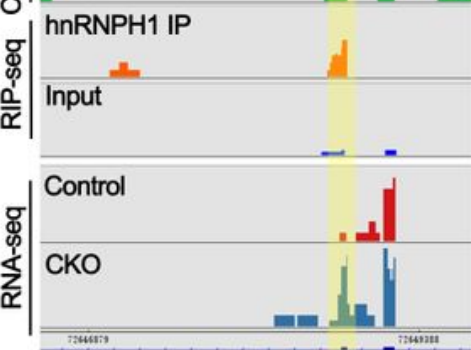

Tcf7/2

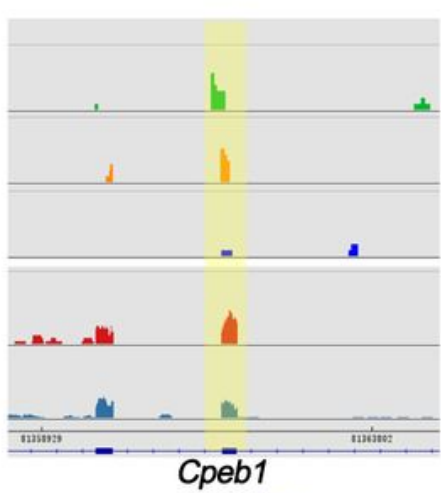

Cpéb1

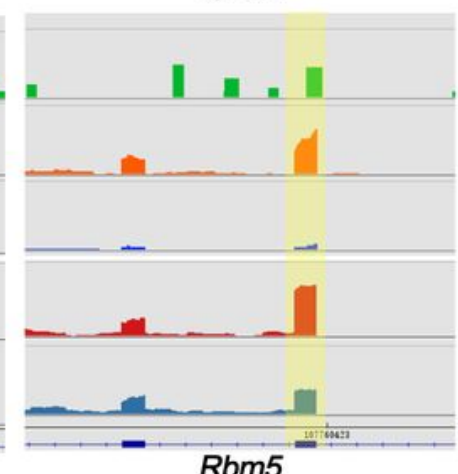

Figure 8

hnRNPH1 cooperates with PTBP2 and SRSF3 to regulate mRNA splicing. (a) Pie-chat shows the summary of binding locations for hnRNPH1 RIP-sequencing (RIP-seq) in spermatogenic cells isolated from P28 WT testes. (b) Top 5 sequence motifs identified from RIP-seq peaks in spermatogenic cells are shown. (c) Venn diagrams showing overlap of hnRNPH1 bound genes and abnormal AS genes (with $\triangle \mathrm{PSI}>0.1$ and $\triangle \mathrm{PSI}<0.1$, respectively) in hnRNPH1 cKO spermatocytes (left) and spermatids (right). 
Histograms show RIP-qPCR analyses of selected mRNA of 11 genes co-precipitated by anti-hnRNPH1, anti-PTBP2, anti-SRSF3 antibodies, and control IgG in RIP experiments performed from purified germ cells. (e) RT-PCR and WB analyses of splicing assays were performed in HEK293T cells transfected with the indicated minigenes and knockdown/overexpression related vectors for hnRNPH1, PTBP2, and SRSF3. (f) Alternative sites in four representative genes bound directly by hnRNPH1, SRSF3, and PTBP2 from RNA-, RIP- and CLIP-seq using IGV software. Splicing sites are indicated by the yellow box.

a

a

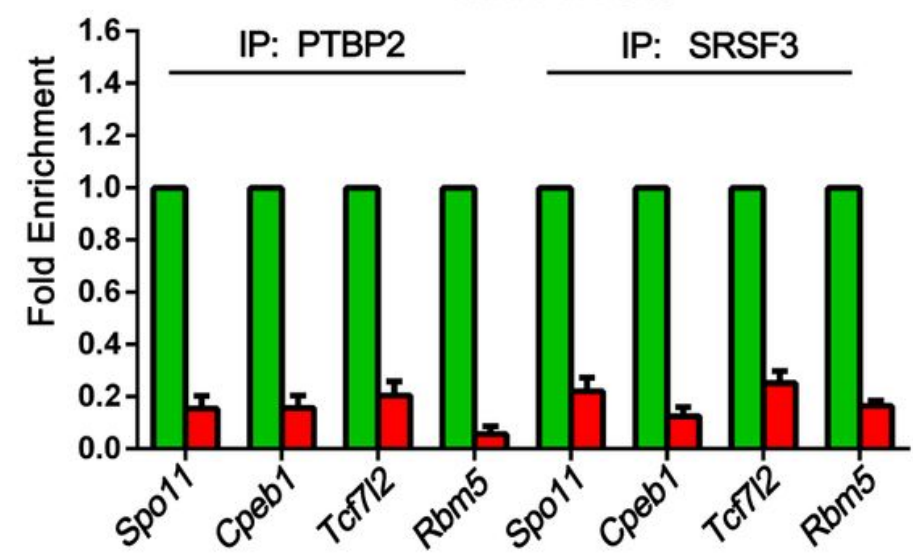

b

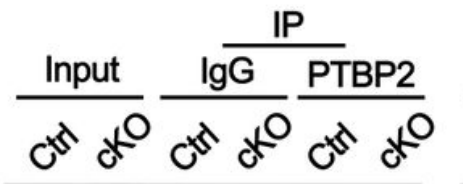

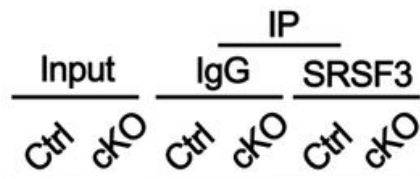
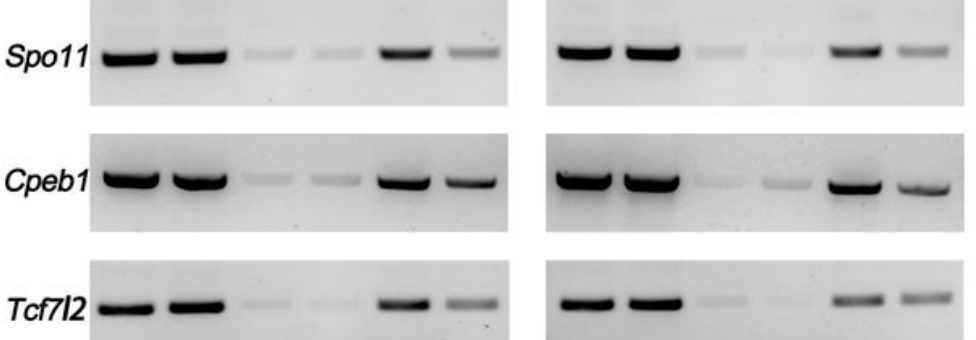

$R b m 5=$

C

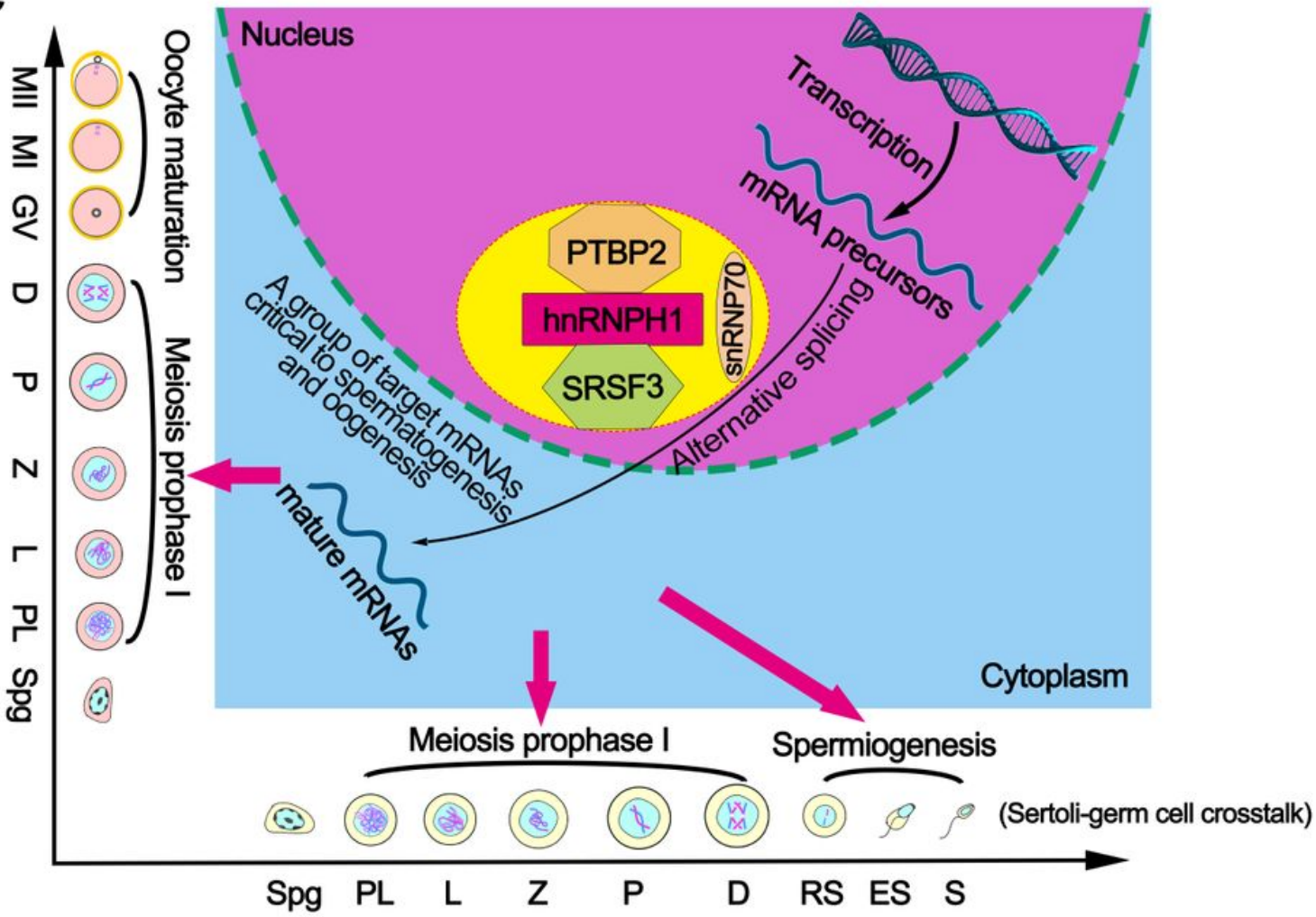

Figure 9

A proposed model of hnRNPH1 function in alternative splicing during spermatogenesis and oogenesis. $(a-b)$ RIP-qPCR (A) and RT-PCR (B) analyses of the association of the selected gene mRNAs with PTPB2 
and SRSF3 in control and hnRNPH1 cKO testis are shown. (c) Schematic model showing that hnRNPH1 binds key splicing factors (e.g., PTBP2, SRSF3) to coordinate proper alternative splicing of its target mRNA transcripts during spermatogenesis or oogenesis.

\section{Supplementary Files}

This is a list of supplementary files associated with this preprint. Click to download.

- SupplementaryTable1IPMS.xls

- SupplementaryTable2spermatocyte.splicingchangedgenes.xls

- SupplementaryTable3spermatids.splicingchangedgenes.xls

- SupplementaryTable4spermatocyte.DEG.xls

- SupplementaryTable5spermatids.DEG.xls

- SupplementaryTable6PTBP2splicinganalyses.xls

- SupplementaryTable7RIPseqofhnRNPH1.xls

- SupplementaryTable8Antibodies.xIsx

- SupplementaryTable9primers.xIsx

- SupplementaryFig.111S.pdf 